\title{
Werkästhetische Voraussetzungen
}

\subsection{Simultane Werkkonstellationen}

Grundsätzlich kann eine simultane Werkkopplung auf zwei verschiedenen Wegen erzeugt werden:

1. Die erste Möglichkeit ist eine nachträgliche, postkompositorische Überlagerung verschiedener Werke jenseits der ursprünglichen Intention der jeweiligen Komponisten. Das simultane Resultat existiert in diesem Fall nicht als kompositorisch ausgearbeitete und festgeschriebene Version. Die Vergleichzeitigung wird erst im Nachhinein vollzogen. Sie kann als interpretative Praxis beschrieben werden, die die sequenzielle Programmabfolge aufbricht. Für dieses Vorgehen soll im Folgenden der Begriff der „Simultanaufführung“ verwendet werden.

2. Die zweite Möglichkeit schließt Werke und Zyklen ein, die mindestens zwei Werke umfassen, die sowohl unabhängig voneinander als auch gleichzeitig aufgeführt werden können. Im Gegensatz zur interpretativen Simultanaufführung ist das Moment der Ver(un)gleichzeitigung hier bereits kompositorisch intendiert und nimmt sowohl Einfluss auf die Struktur und den Aufbau der Werke als auch auf die Form ihrer Verknüpfung. In diesem Fall handelt es sich also um eine kompositorische Praxis, da die optionale Aufführbarkeit durch den Komponisten bereits im Vorfeld antizipiert wurde. In Analogie zur Simultanaufführung wäre der Begriff des Simultanwerks ${ }^{1}$ durchaus geeignet, das Phänomen in seiner Breite zu erfassen. Der Begriff des „Poly-Werks“ ist allerdings mittlerweile auch etabliert. Im Vergleich zum Begriff des Simultanwerks schwingt hier der Verweis auf das qualitative Moment der Verknüpfung von selbstständigen Werkverläufen mit, auf das in dieser Arbeit der Fokus gerichtet ist. Aus diesem Grund wird er im Folgenden von mir auch

1 In der Kunstgeschichte existiert der Begriff des Simultanbilds, das es bereits seit dem Mittelalter gibt. Gemeint ist hier die Zusammenfassung verschiedener zeitlicher und räumlicher Etappen einer Erzählung, die so auf einen Blick erfassbar werden. Analog gibt es im Theater des Spätmittelalters die Simultanbühne, die die verschiedenen Szenen nebeneinander abbildet. Der Zuschauer kann in beiden Fällen selbst wählen, ob er die einzelnen Szenen nacheinander verfolgt oder gleichzeitig überblickt. Die zeitlich chronologische Abfolge entspricht der räumlichen Anordnung. Vgl. Claudia Blümle, „Augenblick oder Gleichzeitigkeit. Zur Simultaneität im Bild“, in: Hubmann/Huss (Hg.), Simultaneität, S. 37-55. 
verwendet. Die von Mahnkopf verwendete Schreibweise „Poly-Werk“ betont das Präfix „Poly“, während die Schreibweise „Polywerk“ (ohne Bindestrich) eher den Werkcharakter hervorhebt. Eine ausführliche Diskussion weiterer Begriffsmöglichkeiten findet sich in Kapitel 2.4. Die Gruppe der komponierten Poly-Werke bildet den Kern meiner Arbeit. Beide Strategien sind miteinander verwandt und Ausdruck der gleichen Fragestellung. Dennoch unterscheiden sie sich in ihrer Genese grundsätzlich voneinander: Das erste Vorgehen ist seinem Wesen nach eine genuine Aufführungspraxis, die ausgewählte Werke in eine simultane Konstellation bringt und auf diese Weise neu kontextualisiert. Da dieser Vorgang postkompositorisch ist, bleiben die Werke in ihrem Kern, ihrer Identität und ihrer Unabhängigkeit davon unberührt. Das zweite Vorgehen ist eine kompositorische Praxis, die bereits im präkompositorischen Prozess ansetzt. Die Werke werden aus der Differenz der verschiedenen Aufführungsoptionen heraus gedacht, mit den formalen, konzeptionellen und qualitativen Konsequenzen für ihr Einzeldasein. Zwar verfügen die Werkstränge über ihren eigenen Abschluss und sind autonom, aber sie sind trotzdem nicht gänzlich unabhängig voneinander entstanden. Auf eine bestimmte Art und Weise sind sie in ihrer Identität zwangsläufig miteinander verbunden und verwandt.

Aus der Perspektive des Rezipienten geben beide Strategien dem Hörer die Möglichkeit einer heterogenen und dezentrierten Hör- und Zeiterfahrung. In seiner Schrift Dauer und Gleichzeitigkeit stellte der Philosoph Henri Bergson fest, dass bei der „instantane[n] Wahrnehmung[...]“ der "Gleichzeitigkeit zweier Prozesse“ die Aufmerksamkeit „sich teilen kann, ohne sich aufzuspalten“. Unteilbarkeit und Teilbarkeit der Aufmerksamkeit fallen zusammen und sind deswegen im Bewusstsein eines Dritten "zugleich einer und zwei“. ${ }^{2}$

„Wenn wir am Ufer eines Flusses sitzen, sind für uns das Vorbeiströmen des Wassers, das Vorübergleiten eines Schiffes oder der Flug eines Vogels, das ununterbrochene Gemurmel unseres tiefen Lebens entweder drei verschiedene Dinge oder ein einziges, ganz wie es uns beliebt.“3

Bei einer simultanen Werkkopplung steht nicht mehr die Entfaltung einer einzigen, sich zeitlich-linear entwickelnden Spur im Fokus der Aufmerksamkeit von Komponist, Interpret und Hörer. Das Hinzufügen einer konkurrierenden Spur, die sich in ihrem Charakter und ihrer formalen Entwicklung anders

2 Henri Bergson: Dauer und Gleichzeitigkeit. Über Einsteins Relativitätstheorie, Hamburg: Philo Fine Arts, 2014, S. 136 f. und S. 366 (Durée et simultaneité. À propos de la théorie de Einstein, Paris: Félix Alcan, 1922).

3 Ebd., S. 137 f. 
verhalten kann, lenkt die Gerichtetheit der Aufmerksamkeit auf den einen Werkverlauf ab. Diese Ablenkung wird in das Werkkonzept integriert. Denn auch wenn in Abhängigkeit von der Besetzung, der Struktur, der Informationsdichte oder der räumlichen Aufstellung die Werke akustisch zurVerschmelzung tendieren können, so ist in den meisten Fällen eine akustische Mehrspurigkeit das Resultat. Diese bietet der Wahrnehmung einen neuen ästhetischen Reiz: Sie stimuliert koordinative Hörfähigkeiten und erschließt ein Feld an neuen Deutungsmöglichkeiten, denn nun treten auch die entstehenden Wechselwirkungen zwischen den Werken in den Vordergrund. Das sich daraus ergebende Verhältnis mutiert nun ebenso zum Gegenstand der Erfahrung und erfährt eine Objektivierung. Der Hörer ist jetzt selbst gefragt, Grenzen zu ziehen und sich zwischen den verschiedenen Angeboten zu entscheiden: $\mathrm{Er}$ kann die Gesamtversion als neue Einheit synthetisieren oder als gebrochenen Zusammenhang auffassen. Er kann die Aufführung als rein absolutes Ereignis hören oder den Verweis auf die anderen Optionen imaginieren. Der größte Reiz wird vermutlich entfaltet, wenn der Hörer einen Abgleich zwischen den verschiedenen akustischen Erscheinungsbildern eines Werkes vornehmen kann und aus der Überfülle des akustischen Informationsflusses heraus ein Prozess der Wiedererkennung, Entschlüsselung und Identifizierbarkeit des Werkobjekts in Gang gesetzt wird. Diese Form eines beweglichen Wahrnehmungsverhaltens führt dazu, dass keine Perspektive mehr das Ganze umfassen kann.

Umberto Eco schrieb 1962 über das offene Kunstwerk:

„[...] jede Ausführung schließlich gibt uns das Werk ganz und befriedigend und gleichzeitig unvollständig, weil sie uns nicht die Gesamtheit der Formen gibt, die das Werk annehmen könnte. ${ }^{4}$

Diese Aussage trifft im Kern auch das Wesen eines Poly-Werks und lässt es als neue Möglichkeit erscheinen, die offene Form zu denken.

Wie in der Einleitung bereits dargestellt, sind zwei Kriterien zur Identifikation eines Poly-Werks ausschlaggebend:

1. Verschiedene Werke können sich in der simultanen Konstellation einer Gruppe von Werken ergänzen beziehungsweise auch umgekehrt aus einer Werkganzheit herausgeeinzelt werden.

2. In den verschiedenen Konstellationen werden die Einzelwerke in ihrer Identität nicht verändert oder nur geringfügig angepasst.

Damit sind bereits die grundlegenden Strukturmerkmale zusammengefasst, die ein Poly-Werk als solches charakterisieren.

4 Eco, Das offene Kunstwerk, S. 49. 


\section{Zur Wandlung und Erweiterung der Werkästhetik im Spannungsfeld von Offenheit und Geschlossenheit}

Das Begriffspaar „offen - geschlossen“ bildet eine zentrale Achse in der Werk- und Formästhetik des 2o. Jahrhunderts. Beide Begriffe rekurrieren auf ein werkästhetisches Spannungsverhältnis, das auf der einen Seite mit einer fixierten Objektgerichtetheit, die reproduzierbar ist, und auf der anderen Seite mit Prozessgerichtetheit, die auch Wandelbarkeit und Nichteindeutigkeit einschließt, assoziiert wird.

Schon aufgrund ihrer immateriellen Medialität haben sich Musik und Komposition in der westlichen Tradition immer in diesem Spannungsfeld bewegt. Zwar hat die Erosion des geschlossenen Werkbegriffs im 2O. Jahrhundert zu einer Vielzahl neuer Sparten geführt, die sich nicht zuletzt auch über den Anspruch entwickelt haben, eine auf Vollendung gerichtete Werkästhetik als ein regressives Modell hinter sich zu lassen. ${ }^{5}$ Im Gegenzug kann jedoch ebenso festgestellt werden, dass das „Werk“ als tradierte, bewährte und fixe Größe weiterhin der Mittelpunkt des zeitgenössischen westlichen Konzertbetriebs ist. Möglicherweise ist der Werkabschluss gerade deshalb für die Komponisten weiterhin attraktiv, weil aus ihrer Sicht die Erfüllbarkeit eines in sich geschlossenen Paradigmas in letzter Konsequenz immer eine Utopie bleiben wird. Denn für den Künstler hat der dynamische Schaffensprozess eine größere Bedeutung als das fertige Produkt. Aus dem Blickwinkel des Komponisten wohnen nahezu jedem „zu Ende gebrachten“ Werk auch andere Möglichkeiten des Abschlusses inne. Umgekehrt präsentiert sich für den Hörer auch das „offenste“ Werk zwangsläufig als fixierte Gestalt. „Wo ein Komponist Möglichkeiten sieht, realisierte neben unterdrückten, sucht der Analysierende nach Notwendigkeit“6, schreibt Carl Dahlhaus 1969. Vor diesem Hintergrund erscheint das Poly-Werk als integratives Werkkonzept, das beide Seiten in sich vereint: einerseits die Notwendigkeit einer finalen Ausformulierung und Präzisierung, die immer auch mit einem Prozess des Verwerfens und des Ausschlusses anderer Möglichkeiten verbunden ist. Andererseits aber auch den Einschluss ausgewählter Möglichkeiten, die Einbindung konträrer Gegenentwürfe, die über eine zufällige Permutation von Elementen - wie sie den offenen Formen der Musik vielfach eingepflanzt wurde - hinausgehen. Dieser

5 Beispielsweise Aktionskunst, Fluxus, Happening, Konzept-, Performance-, Installations- und Klangkunst.

6 Carl Dahlhaus: „Plädoyer für eine romantische Kategorie. Der Begriff des Kunstwerks in der neuesten Musik“, in: ders.: Gesammelte Schriften in zehn Bänden, Bd. 8: 20. Jahrhundert. Historik - Ästhetik - Theorie - Oper - Arnold Schönberg, hg. von Herrmann Danuser u. a., Laaber: Laaber-Verlag, 2005, S. 216-224, hier: S. 224. 
Ansatz erweitert die verbindliche kompositorische Entscheidung um weitere Optionen. Er ist weder radikal negierend noch rein affirmativ und dennoch kritisch und konstruktiv. Wie keine andere Werkform hat das Poly-Werk das Potenzial, auf der formal-organisatorischen Ebene das opus perfectum et absolutum zu spezifizieren, um es im gleichen Atemzug zu differenzieren und zu relativieren. Claus-Steffen Mahnkopf schreibt über das Poly-Werk:

\begin{abstract}
„Damit wird außerdem der Werkbegriff, den das 2o. Jahrhundert immer wieder zu negieren suchte, mit einer neuen Fassung von Identität versehen, denn die Dichotomie von geschlossener und offener Form ist bei einem Poly-Werk überwunden..7
\end{abstract}

Doch was genau meinen Begriffe wie Geschlossenheit und Offenheit? Wie haben sie sich entwickelt und im 20. Jahrhundert möglicherweise selbst eine Wandlung erfahren?

Die Aufwertung des Offenen

Geht man etwas weiter zurück, dann findet sich das Begriffspaar „offen geschlossen" auch bei dem Kunsttheoretiker Heinrich Wölfflin, der es in seinem Hauptwerk Kunstgeschichtliche Grundbegriffe. Das Problem der Stilentwicklung in der neueren Kunst ${ }^{8}$ als sinngleiche Entsprechung der Ausdrücke „tektonisch“ und „atektonisch“ einführt. Es ist eines von fünf Begriffspaaren, die Wölfflin entwickelte, um den „geschlossenen“ klassischen Stil des 16. Jahrhunderts vom „offenen“ barocken Stil des 17. Jahrhunderts zu unterscheiden. Wölfflins Untersuchungen beziehen sich auf die Malerei, die Plastik und die Architektur und gehen von der Analyse des Bildaufbaus und der Komposition auf der formalen Ebene aus. Während beispielsweise der klare Aufbau einer klassischen Komposition im Einklang mit der rechtwinkligen begrenzenden Bildrahmung steht - den vertikalen und horizontalen Bildachsen entsprechend -, wird diese strenge Bildgliederung im Barock aufgelöst und die Diagonale der Figuren in ein Spannungsverhältnis zum Bildrahmen gesetzt.

Wölfflin stellt die Darstellung „einer in sich selbst begrenzten Erscheinung [...], die überall auf sich selbst zurückdeutet“, dem Stil der offenen Form gegenüber, die „überall über sich selbst hinausweist, unbegrenzt erscheinen will, obwohl eine heimliche Begrenzung immerfort da ist und eben den Charakter der Geschlossenheit im ästhetischen Sinne möglich macht“ ${ }^{9}$ Die

7 Claus-Steffen Mahnkopf: Kritik der neuen Musik. Entwurf einer Musik des 21. Jahrhunderts, Kassel: Bärenreiter, 1998, S. 118.

8 Wölfflin: Kunstgeschichtliche Grundbegriffe, S. 147.

9 Ebd. 
offene Form ist in Wölfflins Darstellung eine Spielart, eine Aufweichung des Geschlossenen, eine Überführung des Stabilen, Gewollten, Starren und Begrenzten ins Fließende, Zufällige, Ungewollte oder Unendliche. Nicht nur das Bedürfnis nach „Ordnung, Gesetz, Rahmung und Festigkeit, Dauerhaftigkeit" hat seine Daseinsberechtigung, sondern ebenso das "Nichtgewollte, Nichtgeschlossene, das Zufällige, Vorübergehende und Unbegrenzte“. Die "Werte des Seins“ werden den „Werte[n] der Veränderung“ gegenübergestellt. ${ }^{10}$ Auch ob etwas "gewollt erscheint oder nicht" ${ }^{\text {"11 }}$, scheint ihm bereits ein entscheidendes Unterscheidungsmerkmal zu sein. Mit seiner Kategorisierung nimmt Wölfflin vermutlich erstmals eine Aufwertung der Kategorie des „Nichtgeschlossenen“ - die bis zu diesem Zeitpunkt wohl nie konkret benannt wurde - vor. ${ }^{12}$

Auch Umberto Eco - der als erste „bewußte“ Poetik des offenen Kunstwerks den Symbolismus in der zweiten Hälfte des 19. Jahrhunderts ausmach ${ }^{13}$ - stellt in seinem viel rezipierten Hauptwerk Das offene Kunstwerk die „statische und unmißverständliche Bestimmtheit der klassischen Renaissanceform "14 der „offenen Form“ des Barock gegenüber, die dem zentrierten Renaissanceraum durch ein Auflösungsstreben entgegengesetzt ist. Durch ihre dynamische Form bewirkt sie beim Betrachter eine bewegliche und flexible Rezeptionshaltung, eine analog zur Unbeständigkeit von Kunst, Wissenschaft und Welt multiperspektivische Sichtweise. ${ }^{15}$

„[...] das Streben nach Bewegung und Illusion bewirkt, daß die plastischen Massen des Barock niemals die Feststellung eines bevorzugten, frontalen, definiten Standpunktes gestatten, von dem aus sie zu betrachten wären, sondern den Betrachter ständig dazu veranlassen, den Standort zu wechseln, um das Werk unter immer neuen Aspekten zu sehen, so als ob es in beständiger Umwandlung begriffen wäre."16

\footnotetext{
$10 \quad$ Ebd., S. 159.

11 Ebd., S. 149.

12 Neben dem Barock lassen sich auch andere Parallelen zu offenen Formbildungen finden, wie Hans-Ulrich Reck beispielsweise anhand der Alhambra zeigt. Vgl. hierzu Hans Ulrich Reck: Grenzziehungen. Ästhetiken in aktuellen Kulturtheorien, Würzburg: Königshausen und Neumann, 1991, S. 158-159. Werner Hofmann wiederum zieht Parallelen zwischen einem „polyfokalen“ Mittelalter und einer „polyfokalen“ Moderne. Er grenzt diese von der „monofokalen“ Renaissanceform ab, denn „sie verlangt vom Betrachter kein wanderndes Fokussieren, d. h. kein Umschalten der Wahrnehmung von einem Fokus zum anderen“ (Werner Hofmann: Die Moderne im Rückspiegel. Hauptwege der Kunstgeschichte, München: Beck, 1998, S. 16-17).

13 Eco, Das offene Kunstwerk, S. 36.

14 Ebd., S. 34.

15 Siehe ebd., S. 35.

16 Ebd.
} 
Offene Formen - Geschlossene Werke

In der Neuen Musik vermittelt der Begriff des geschlossenen Werks die Idee eines in sich stimmigen, abgrenzbaren und reproduzierbaren Objekts. Die Kritik an einer Ästhetik des Werkabschlusses ist dabei vor allem eine Kritik an einem kohärenten und konsistenten Formbegriff. Form als primäre Kategorie der Komposition ist eine Errungenschaft der klassischen Instrumentalmusik und gleichermaßen ihr Relikt. Ihre Ineinssetzung mit dem Werkbegriff bedeutet allerdings die missverständliche Vermischung zweier verschiedener Ebenen. ${ }^{17}$ Zu den werkkonstituierenden Eigenschaften zählen zuallererst Autorschaft, Individualität, Originalität, Fixierung, Handelbarkeit, Reproduzierbarkeit und ein vom Autor unabhängiges Eigenleben. Einheitlichkeit, Eindeutigkeit und Vollendung sind dagegen Merkmale einer formalen Physiognomie. Die Formebene ist aber eine der Werkebene nachgeordnete Kategorie, denn die Werkebene ist in ihrem Wirkungs- und Bedeutungsfeld weitreichender, schließt die postkompositorischen Prozesse mit ein und geht über die rein komponierte Form eines Musikstückes hinaus. Auch da, wo Form keine primäre Kategorie mehr oder Musik vermeintlich „formlos“ ist, gibt sie ihren werkhaften Geschlossenheitscharakter nur graduell, nie vollständig auf. Die allgemeinen oben genannten Werkeigenschaften bleiben erhalten. Auch ein geschlossenes Werk kann eine offene Form besitzen.

Die Überführung der Kategorie des „Offenen“ von einem interpretatorischen Spielraum - der sich aus dem Zusammentreffen von Werkobjekt und individueller Rezeption ergibt - zu einem "produktiven Programm“ wird von Eco mittels einiger Werkbeispiele europäischer Komponisten der Darmstädter Schule wie Klavierstück XI von Karlheinz Stockhausen, Scambi von Henri Pousseur, 3. Klaviersonate von Pierre Boulez oder Sequenza per flauto solo von Luciano Berio illustriert. ${ }^{18}$ Der als offen, mobil oder auch variabel bezeichneten Form in der Musik kam ab der Mitte des 20. Jahrhunderts bis ungefähr in die 8oer Jahre eine essenzielle Bedeutung zu. Sie wurde gleichgesetzt mit Spontanität, Indetermination oder Zufall und Werken zugeschrieben, in denen der eindeutige, normative Formverlauf durch ein Möglichkeitsfeld verschiedener Formabschnitte, Module, Partiturseiten, Formstränge etc. mit oder ohne Regeln ersetzt wurde und der Interpret bestenfalls während der Ausführung eine Auswahl traf. Der Entscheidungsspielraum des Interpreten wurde auch auf andere Organisationsebenen (beispielsweise Tempi, Tonhöhen, Dauern, Notation etc.) ausgedehnt und außerdem die Möglichkeit des Improvisierens eingebaut. Dadurch floss ein Moment von Unvorhersehbarkeit

\footnotetext{
17 Eine konzentrierte Zusammenfassung der Problematik der Gleichsetzung von Form und Werk bietet Holzer: Zur Kategorie der Form in neuer Musik, S. 53-59. 
und Variabilität in die Werkbildung ein. Aus der Utopie des fixierten "Seins“ wurde ein potenziell fortlaufendes „Werden“. Das Werk verfügte nun nicht mehr über einen eindeutigen vorbestimmten und geschlossenen Ablauf, aber dafür über mehrere Ausführungsmöglichkeiten in Bezug auf ausgewählte Parameter. Der Zeitpunkt ihrer definitiven Auswahl lag nicht mehr innerhalb der Kompositionsphase. Sie fiel idealerweise mit dem Zeitpunkt der Ausführung zusammen und erweiterte dadurch scheinbar den Interpretationsradius. Grundsätzlich schien Offenheit eine Freiheit zu sein, die sich vor allem in einem vom Komponisten festgelegten Rahmen bewegte und damit vor allem die Formalisierung jener Freiheit bedeutete. So spricht Boulez selbst von der Notwendigkeit einer vom Komponisten kontrollierten, dirigierten und geplanten interpretatorischen Freiheit. ${ }^{19}$ Die „bausatzartige ${ }^{20}$ Offenheit der Komponisten der Darmstädter Schule minderte weder den kompositorischen Gestaltungsanteil- und aufwand noch den konventionellen geschlossenen Werkcharakter. Auch löste sie die Hierarchie zwischen Komponist und Interpret nicht auf. Diese wurde dagegen umso mehr von John Cage infrage gestellt, der sich für eine Indetermination jeglicher musikalischer Kategorien und - im Gegensatz zu Boulez - für eine Aufgabe kompositorischer Kontrolle aussprach.

\subsection{Die Simultanaufführung bei John Cage}

\subsubsection{Leere versus Überfluss}

„Daß zwei oder / mehrere Dinge / gleichzeitig geschehn / ist ihre Beziehung." ${ }^{21}$

(John Cage)

In dem Stück 4'33" aus dem Jahre 1952 von John Cage (1912-1992) wird das komponierte Werk zurückgenommen. An die Stelle eines aufführbaren Werks tritt die Stille als leeres Werk. Das „Werk“ fungiert in erster Linie nur noch als Rahmensetzung, um die Aufmerksamkeit des Hörers für die äußeren Geräusche der Umwelt, die jenseits einer subjektiven und gestaltenden Intention eines Autors existieren, empfänglich zu machen. Anstatt diese

19 Siehe Pierre Boulez, Alea, 1957, nachzulesen in: Rainer Nonnenmann (Hg.): Mit Nachdruck. Texte der Darmstädter Ferienkurse für Neue Musik, Mainz: Schott, 2010, S. 173-186.

$20 \quad$ Siehe hierzu auch Carl Dahlhaus in Thomas (Hg.): Form in der Neuen Musik, S. 74.

21 John Cage, 45' für einen Sprecher (1954), in: ders.: Silence, hg. von Ernst Jandl, 9. Aufl., Frankfurt am Main: Suhrkamp, 2017, S. 85 f. 
Klänge zu unterdrücken oder (un)bewusst auszublenden, sollen sie in den Mittelpunkt des Bewusstseins rücken und zur neuen klanglichen Erfahrung mutieren. Für Cage gibt es keine Stille an sich. „[...] it is full of sound, but sounds which I did not think of beforehand, which I hear for the first time the same time others hear.“22 Damit wird die - bis dahin eindeutige - Trennung zwischen den Klängen des Werks und den Klängen der Welt aufgehoben.

4'33" ist nicht unbedingt an den räumlichen Rahmen einer Konzertaufführung gebunden. Allein dass ein Mensch an einem beliebigen Ort, zu beliebiger Zeit eine Rezeptionshaltung einnimmt und sich für eine beliebige Dauer auf die Geräusche und Klänge des ihn umgebenden Ortes als erweiterte Klangerfahrung einlässt, stellt nach Cage eine Aufführung von 4'33" dar. Ist die Stille nach Cage also nicht steril, sondern ein lebendiger und fruchtbarer Raum des Erlebbar-Machens wahrnehmbarer Klang- und Geräuschereignisse, die unabhängig von einer gestaltenden kompositorischen Intention existieren, dann könnte konsequenterweise bereits jegliche Werkaufführung als verschränkte Simultanaufführung eines „Werks" und seiner „Umgebung“ gedacht werden. ${ }^{23}$

Die extreme Reduktion der musikalischen Werkidee, die Cage im Alter von vierzig Jahren vollzog, steht einer entgegengesetzten Tendenz in seinem Gesamtschaffen gegenüber: der gleichzeitigen Aufführung verschiedener Werke. Sein größtes Orchesterwerk 108 aus dem Jahre 1991 ist gleichzeitig sein letztes großes Werk, das auch mit zwei anderen musikalischen Werken simultan aufgeführt werden kann. ${ }^{24}$ Seine zeitliche Dauer von 43:30 Minuten ist wohl kein zufälliger Verweis auf 4'33". Beide Werke markieren den zeitlichen Anfangs- und Endpunkt einer Entwicklung im Schaffen von Cage, die Stille und Klang, Stille und Werk sowie Werk und Werk immer wieder miteinander verschränkt. So steht der leere Zustand der Stille in 4'33" einer bisweilen massenhaften Akkumulation verschiedenster Werke gegenüber, wie sie beispielsweise in A House Full of Music im Bremer Überseemuseum am 10. Mai 1982 realisiert wurde: Innerhalb von neunzig Minuten wurden ca. 15 o verschiedene Stücke verschiedenster Komponisten und Traditionen aus verschiedenen Zeiten

22 Zitiert aus einem Brief an Helen Wolff in John Cage: „Es wird niemals Stille geben ..., Ein bisher unveröffentlichter Briefwechsel zwischen Helen Wolff und John Cage“, in: MusikTexte. Zeitschrift für Neue Musik 106, 2005, S. 47-5o, hier: S. 48.

23 Ebenso könnte jene "Stille“ auch als Verweis auf das eigene innere Bewusstsein des Hörers gedeutet werden, das während einer Konzertaufführung ebenso unabhängig wie unwillentlich auf die eigenen Gedankengänge gerichtet sein kann, wodurch sich beide „Spuren“ in der Aufmerksamkeit miteinander vermischen.

24108 ist sowohl mit $O n e^{8}$ für Cello mit Rundbogen als Cellokonzert 1oga, mit One ${ }^{9}$ für Shō als 1ogb und mit $T w o^{3}$ für Shō und Conch Shells als Doppelkonzert 110 aufführbar. 
überlappend und simultan durch ca. 8oo Kinder und Jugendliche aufgeführt. ${ }^{25}$ Diese Aufführung wurde darüber hinaus als Radiostück weltweit übertragen. Für den Radiohörer mündete das Spektakel, das vor allem eine rein quantitative Schichtung verschiedenster Werke aus verschiedensten Epochen war, vermutlich in eine schwer zu durchdringende Klangtotalität.

Geräuschhafte Stille und klanglicher Überfluss markieren die grundlegenden Achsen zweier Tendenzen, die im Gesamtwerk von Cage auch in den dazwischenliegenden Abstufungen ihren Ausdruck finden. Während die geräuschhafte Stille auf eine Befreiung des Klanges von der Intention eines Autors zielt, ist die Massenaufführung auf eine Befreiung des Werks aus der Klammer der idealerweise sterilen "Stille“ des abgeschlossenen Konzertraumes gerichtet. In beiden Fällen findet eine situative Neukontextualisierung statt. „Stille“ fungiert nicht mehr nur als hintergründige Rahmenbedingung, die es dem Hörer ermöglicht, seine Aufmerksamkeit für eine bestimmte Dauer, auf eine einzige, sich linear entfaltende musikalische Spur zu bündeln. Indem die sekundären Störgeräusche eine ästhetische Aufwertung erfahren, emanzipieren sie sich als eigenständige Ebene mit einer eigenen klanglichen Qualität. Folglich mutiert nach Cage jegliches klangliches Umgebungsgeschehen, das eine Werkaufführung begleitet, zu einer zusätzlichen, gleichwertigen Dimension. Die entstehenden Wechselwirkungen entziehen sich wiederum der Kontrolle des Autors.

„Wenn die Musik Umweltgeräusche zulassen kann, ohne davon beeinträchtigt zu werden, so handelt es sich um moderne Musik“26, gibt John Cage als Kriterium für gelungene moderne Musikwerke an. In der Juillard Lecture, die Cage 1952 vor Studenten der Juillard School of Music hielt und die zeitgleich mit der Aufführung von Klavierwerken von Morton Feldman, Christian Wolff und John Cage durch den Pianisten David Tudor stattfand, wird eine Episode über Christian Wolff zum Ausgangspunkt der Deutung von Simultaneität als erweiterter Hörerfahrung, die unabhängig voneinander existierende Klänge und Ereignisse als gleichwertig anerkennt: Während Wolff ein eigenes Klavierstück spielte, das auch Pausen der Stille in sich trug, drangen die Geräusche der Straße durch ein offenes Fenster in den Raum und übertönten bisweilen sein Spiel. Nachdem das Stück zu Ende war, schloss ein Freund Christian Wolffs, der als Hörer anwesend war, die Fenster und fragte, ob er das Stück nun noch

25 Für eine detaillierte Beschreibung der Aufführung siehe auch Philipp Schäffler: Die Idee der Bildung im Schaffen von John Cage, Mainz: Schott, 2009, S. 182-217.

26 Richard Kostelanetz:John Cage im Gespräch. Zu Musik, Kunst und geistigen Fragen unserer Zeit, Köln: DuMont, 1989, S. 162. 
einmal ohne Störgeräusche hören dürfe. Wolff klärte ihn daraufhin auf, dass die zufällig eindringenden Geräusche keine Störung gewesen seien. ${ }^{27}$

Die Trennung von Alltagsgeräuschen und musikalischem Werk und damit von Alltag/Leben und Kunst verliert ihre Eindeutigkeit. Scheinbar zufällig auftretende akustische Umgebungsgeräusche während einer Aufführung sind in ihrer Unvorhersehbarkeit nicht als Störung oder Ablenkung aufzufassen, sondern als gleichwertige klangliche Erfahrung. Die zufälligen Reize sollen in die kontrollierte und auf die Musik gerichtete Aufmerksamkeit des Hörers integriert werden. Cage passt sich in seiner kompositorischen Praxis seinen Beobachtungen klanglicher Umgebungsgeräusche strukturell an. Durch die Einbindung geräuschhafter Klänge oder auch durch Ausschaltung der eigenen subjektiven Intention mittels objektivierender Zufallsstrategien, der Befragung des I Ging etc. wird die Schaffung eines Klangbildes ermöglicht, das seine enge Bindung an die persönliche und selbstbezogene Imagination des Autors auflöst und sich in der Unbestimmtheit des Resultats der peripheren klanglichen Außenwelt annähert und zuwendet. Es findet eine qualitative Öffnung füreinander statt, die die Kluft zwischen Werk/Kunst und Alltag/ Leben aufweicht. ${ }^{28}$

Die Aufweichung der traditionell den musikalischen Werkcharakter mitkonstituierenden Werkgrenzen findet sich bei Cage auf verschiedenen Ebenen wieder. Die Aufhebung der Trennung zwischen Kunst und Leben, Musik und Alltagsgeräusch oder zwischen Komponist, Interpret und Hörer wird ebenso propagiert wie eine Aufhebung der Trennung zwischen den Künsten, wie sie beispielsweise auch in der Koexistenz von Musik und gesprochenem Vortrag in der Juillard Lecture der Fall ist. ${ }^{29}$ Handelte es sich hier aber um eindeutig

27 John Cage, Juillard Lecture, in: ders.: A Year from Monday. New Lectures and Writings by John Cage, London: Boyars, 1985, S. 101.

28 Versteht man die Schließung eines Kunstwerks aber nicht nur in einem qualitativen Sinn, sondern bezieht sie ebenso auf den zeitlichen Rahmen, so verweisen umgekehrt gerade die Titel vieler Cage-Stücke auf fixe zeitliche Außengrenzen, die die Dauer einer Aufführung übergenau determinieren und formalisieren. Beispielsweise sind die Titel 3'57.9864" für Klavier und 34'46.776" für Klavier auch gleichzeitig die Dauern der Stücke. Beide können gleichzeitig mit 45' für einen Sprecher aufgeführt werden, wobei 45' ebenfalls eine Dauernangabe ist. Auch die Zahl der Aufführenden findet sich in den späten Werken der Number Pieces als Titel wieder und gibt eine klare Begrenzung der Zahl der aufführenden Musiker vor. So besteht das bereits erwähnte Orchesterwerk 108 auch aus 108 Aufführenden. Wenn es zeitgleich mit $O n e^{8}$ (als achtes Solostück innerhalb der Number Pieces) für einen Cellisten aufgeführt wird, resultiert daraus 109.

29 Das bekannteste Beispiel ist zweifellos das Black Mountain Happening aus dem Jahre 1952. Die Zahl der Mitwirkenden und die Dauer des Ereignisses sind unbestimmt, und die verschiedenen Genres Musik, Tanz, Poesie, Film etc. werden frei kombiniert. An der Aufführung im Sommer $195^{2}$ waren John Cage, Mary Caroline Richards, Charles Olson, 
voneinander unterscheidbare und abgrenzbare Wahrnehmungsmodi, können in den rein musikalischen Simultanaufführungen die Komponenten einander auf andere Weise durchdringen. Diese Praxis findet sich in Cages Schaffen in dem langen Zeitraum von 1953 bis 1991, seiner gesamten zweiten Lebenshälfte.

\title{
1.3.2 Simultane Aufführungsformen bei Cage
}

\begin{abstract}
„In Sevilla bemerkte ich an einer Straßenecke die Vielfalt von gleichzeitig sichtbaren und hörbaren Ereignissen, die alle in der Erfahrung zusammenlaufen und mir gefallen. Für mich war es der Anfang von Theater und Zirkus.“30
\end{abstract}

(John Cage)

John Cage kann als der einzige Komponist gelten, der seine Werke mit einer gewissen Konsequenz simultan aufführen ließ. Die Simultanaufführung war bei ihm kein beiläufiges Ereignis. Sie ist Teil einer Praxis, die sich aus der Haltung speist, den Werkcharakter - im Hinblick auf Abgeschlossenheit und Vollendung - auf der Ebene der Komposition aufzuheben und stattdessen eine Verschiebung dieser Kategorie in Richtung der Aufführung und der Rezeption zu erreichen. Die Werksimultaneisierungen sind daher auch nie schriftlich und werkhaft fixiert. Vielmehr handelt es sich um eine Aufführungspraxis. Das schriftlich Gegebene wird neu kontextualisiert, indem durch die Gegenwart anderer Werke neue akustische Umgebungsbedingungen geschaffen werden.

Die Liste der simultan aufführbaren Werke bei Cage ist lang und umfasst mehr als fünfzig seiner Werke. Es existieren mehrere Stufen der Simultaneisierung, die von einer sehr freien und beliebigen Kombination bis zu einer konkreteren Festlegung reichen. Insgesamt können vier Gruppen unterschieden werden:

1. Freie Kombination von Repertoirestücken

Die freieste und offenste Form der Werkschichtung ist zweifellos die beliebige Kombination beliebiger Repertoirestücke durch eine beliebige Anzahl von Spielern. Dabei handelt es sich um von Cage organisierte Events und Happenings, die unter Namen wie Musicircus (1967), Musicircus for Children (1984), A House Full of Music oder Scottish Circus (1990) stattfanden. Nach dem Motto „Mehr ist mehr“ wird pure Quantität zur entscheidenden Voraussetzung eines erfolgreichen Spektakels: Es gilt,

Nicholas Chernovitsch, Richard Buckminster Fuller, Merce Cunningham und Robert Rauschenberg beteiligt.

$3^{\circ}$ John Cage: „From Where'm Now‘. Eine autobiographische Skizze“, in: MusikTexte. Zeitschrift für Neue Musik 106, 2005, S. 21-26, hier: S. 21. 
möglichst viele Teilnehmer zu versammeln, um ein möglichst dichtes, komplexes und chaotisches Klangbild zu erzeugen. Zusätzlich wird durch den Eklektizismus von Repertoirestücken verschiedenster Epochen und Kulturen die "gesamte Musikgeschichte zum ,Materialfundus' da die einzelnen wie auch immer gearteten Musikstücke aus dem historischen Zusammenhang gelöst und so behandelt [werden], als wären sie Klänge oder Geräusche“31. Die Simultaneisierung verschiedener Musikstücke geht hier mit der Simultaneisierung verschiedener historischer Zeiten einher.

2. Freie Kombination einzelner simultaner Stimmen/Parts eines Werks In Werken wie Music for Piano $\left(1953^{-1956}\right)^{32}$, Radio Music $(1956)^{33}$, dem Concert for Piano and Orchestra (1957/58), Atlas Eclipticalis (1961-1962), HPSCHD (1967-1969) und Hymnkus (1967-1969) können die einzelnen instrumentalen, elektronischen oder radiophonischen Parts vom Solo bis zur vollen Instrumentation in allen denkbaren Besetzungen miteinander kombiniert werden. Damit ändert sich die Werkgestalt mit jeder Aufführung. Konzeptionell sind also alle Stimmen autonom, in der Ungleichzeitigkeit, aufführbar. Ihnen wird das Potenzial zugestanden, eine individuelle Aufführung auszufüllen oder sich mit einer anderen Stimme zu verbinden. Die Werke sind zumeist in Stimmen notiert, statt in Form einer Partitur. Die komplette Werkentität kann also nur gehört und nicht gelesen werden.

Bereits im Concert for Piano and Orchestra entfällt die Partitur als vertikale Organisationsform. Der ursprünglichen Intention eines Orchesterapparats entgegengesetzt, sind alle Stimmen Solostimmen, die beliebig simultan kombiniert werden können und eigene, typischerweise unspezifische Titel (beispielsweise Solo for Flute) erhalten. Auch eine Aufführung aller Orchesterstimmen ohne Klavier ist denkbar, womit es dann kein Klavierkonzert mehr wäre. Jeder Spieler trägt selbst die Verantwortung für seine Entscheidungen und entwickelt individuell einen Plan zur

31 Schäffler: Die Idee der Bildung im Schaffen von John Cage, S. 183 f.

32 Siehe hierzu die Dissertation von Martin Erdmann: Untersuchungen zum Gesamtwerk von John Cage, Diss. Bonn, 1993, S. 62 f.

33 Das Radio als Medium eines simultanen Zeitkonzepts ist in Cages Schaffen mehrfach vorhanden und verweist auf die Bedeutung der Simultaneität in seinem Denken. Vgl. hierzu Alana Pagnutti: Reception. The Radio-Works of Robert Rauschenberg and John Cage, Brooklyn: Smith + Brown, 2016. Ebenso sei hier auf die Radioarbeit Solitude Trilogy von Glenn Glould hingewiesen. Das Stück vereint drei einzelne Dokumentarfilme, die zwischen 1967 und 1977 entstanden sind. Gould legt die verschiedenen Stimmen aus den Interviews teilweise übereinander. 
Umsetzung der notierten Stimme. Der in der Gesamtbesetzung eingesetzte Dirigent entwickelt keine synchronisierende und koordinierende Kraft, sondern gibt nur noch rotierende Sekundbewegungen vor und eine Gesamtdauer. Heinz-Klaus Metzger sieht darin ein "positives Modell von Anarchie" und bezeichnet solche flexiblen Aufführungsformen mehrfach als „kollektives Modell von Individualanarchie“. ${ }^{34}$ Die soziale Dimension dieses kompositorischen Vorgehens wird von Cage selbst in verschiedenen Schriften und Interviews immer wieder thematisiert. Er sieht sich selbst einem zu Anarchie tendierenden Gesellschaftsmodell verbunden: „I'm interested in society, not for purposes of power, but for purposes of cooperation and enjoyment." ${ }^{\text {"3 }}$

3. Simultane Überlagerung sukzessiv angeordneter Stücke innerhalb einer Werkentität

Die freie Schichtung der Instrumentalebenen findet ihre Entsprechung in der offen gehaltenen Reihung oder der überlappenden oder gleichzeitigen Überlagerung des Materials, der Seiten- und der Stückreihenfolge. So in Quartet (Percussion Quartet, 1935), in She is Asleep (1943, für Stimme und präpariertes Klavier), in Music for Piano (1953-1956), in Winter Music (1957, 1-2o Pianisten), im Concert for Piano and Orchestra (1957/58), in den Song Books (1970) oder auch in Twenty-Eight, TwentySix and Twenty-Nine (1991). Beispielsweise sind die Song Books eine Sammlung vokaler Stücke, die notationell sukzessiv aneinandergereiht sind, aber beliebig und auch simultan miteinander kombiniert werden können. Analog zu Punkt 2 ändert sich durch die vielfältigen inwendigen kombinatorischen Möglichkeiten innerhalb des von Cage gesetzten Rahmens die äußere Werkgestalt mit jeder Aufführung. Werden ganze Werkentitäten miteinander simultan kombiniert, setzt sich dieser Prozess über die gesetzten Werkrahmen fort.

4. Konkrete Simultaneisierung einzelner Werke

Ab 1953 beginnt Cage, Werke aufzuaddieren. ${ }^{36}$ Wie bereits dargestellt, ist dieser Prozess bei ihm primär eine Aufführungspraxis, da die Simultaneisierung nicht notationell festgelegt und damit werkhaft

34 Heinz-Klaus Metzger: Die freigelassene Musik. Schriften zu John Cage, hg. von Rainer Riehn u. a., Wien: Klever, 2012, S. 97.

35 Richard Kostelanetz: Conversing with Cage, New York/London: Routledge, 2003, S. 274.

36 Neben der Akkumulation seiner eigenen Werke ist auch die Kombination mit Werken anderer Komponisten denkbar. 1977 kombinierte Cage Address mit der Musik von Erik Satie. Auch gibt es die bereits erwähnte Gleichzeitigkeit verschiedener Künste im Sinne einer Aufhebung der Trennung zwischen ihnen. Ein Beispiel ist hier auch das letzte Orchesterwerk 103, das mit dem Film One ${ }^{1 l}$ addiert werden kann. 
fixiert oder technisch synchronisiert ist. Prinzipiell ist jedes Werk mit jedem kombinierbar. Je nach Gelegenheit wird daraus ein „Musicircus“37 oder eine im Vorfeld konkret geplante Kombination eigener Werke. Die folgende Liste erhebt somit auch keinen Anspruch auf Vollständigkeit. Diese wäre im Sinne von Cage sicher auch nicht möglich. Es handelt sich um eine Auswahl, die auch einen Eindruck vermittelt wie Cage Werke kombiniert hat, die von ihrer Entstehungszeit eher nah beieinander liegen. Die Zeiträume umfassen zumeist zwei bis drei Jahre. Der größte Entstehungsabstand ist mit dreizehn Jahren zwischen Winter Music (1957) und den Song Books (1970) zu finden. Es ist anzunehmen, dass noch größere zeitliche Abstände zwischen den Werken prinzipiell keinen Hinderungsgrund für eine Kombination darstellen würden.

Beispiele für die von Cage intendierten Möglichkeiten der Werkkombination - 26’.1499" (für einen Streicher, 1953-1955) + 27'10.554" (für einen Schlagzeuger, 1956), 31'57.9864“ (für einen Pianisten, 1954), 34'46.776” (für einen Pianisten, 1954) oder $45^{\prime}$ (für einen Sprecher, 1954)

- 31'57.9864" (für einen Pianisten, 1954) + 45' (Sprecher, 1954)

- 31'57.9864" (für einen Pianisten, 1954) + 34'46.776" (für einen Pianisten, 1954)

- 34'46.776" (für einen Pianisten, 1954) + 45' (Sprecher, 1954)

- Winter Music (1-20 Pianisten, 1957) + Atlas Eclipticalis (variables Ensemble, 1-98 Orchestermusiker, 1961/62, Instrumente können elektronisch verstärkt und nach der grafischen Notation von Cartridge Music ausgesteuert werden)

- Aria (Stimme, 1958) + Fontana Mix (Tonband, 1958) ${ }^{38}$ oder Teile des Concert for Piano and Orchestra (1957/58)

- Solo for Voice 2 (Solostimme, 1960) + Teile des Concert for Piano and Orchestra (1957/58), Fontana Mix (Tonband, 1958) oder Cartridge Music (variables Ensemble, 1960)

- Concert for Piano and Orchestra (1957/58) + Rozart Mix (Tape, 1965) + Song Books (Solostimme, 1970)

37 Auf die Frage von Daniel Charles, was sich Cage unter einer „Zirkus-Situation“ vorstelle, antwortete Cage: „Der Prozeß öffnet sich, um Dinge einzubeziehen, die keine emotionalen Eigenschaften besitzen, aber ebenso, um Objekte wiedereinzubeziehen, die durch Bedeutung und Zweck belastet sind. Diese Objekte werden innerhalb des Prozesses mitgetragen, sie dominieren ihn aber nicht mehr und verwandeln ihn nicht in ein Objekt.“ (John Cage: Für die Vögel. Gespräche mit Daniel Charles, hg. von Daniel Charles, Berlin: Merve, 1984, S. 179.).

38 Zur Kombination von Aria + Fontana Mix siehe auch Juan Parra Cancino: „Historical contextualization in live electronic music. Reconstructing Aria/Fontana Mix by John Cage", in: ders. u. a. (Hg.): Metacage. Essays on and around Freeman Etudes, Fontana Mix, Aria, Leuven: Leuven University Press, 2009, S. 17-42. 
- Concert for Piano and Orchestra (1957/58) + Aria + Solo for Voice I, Fontana Mix, Indeterminacy, Solo for Voice II, WBAI, Song Books

- Fontana Mix (Tonband, 1958) + Teile des Concert for Piano and Orchestra, Aria (Stimme, 1958), Solo for Voice 2 (Solostimme, 1960) und/oder Song Books (Solostimme, 1970)

- Winter Music (1-20 Pianisten, 1957) + Atlas Eclipticalis (variables Ensemble, 1961/62) oder Song Books (Solostimme, 1970)

- Atlas Eclipticalis (variables Ensemble, 1961/62) + Song Books (Solostimme, 1970)

- Concert for Piano and Orchestra (1957/58), + Atlas Eclipticalis (variables Ensemble, 1961/62)

- Atlas Eclipticalis (Schlagzeugteile, 1961/62) + Rozart Mix (Tape, 1965) + Song Books (Solostimme, 1970)

- Song No. 85 (Stimme, 1970) + Branches (variables Ensemble, Pflanzenmaterial, 1976)

- Child of Tree (Percussion solo, Pflanzenmaterial, 1975) + Branches (variables Ensemble, Pflanzenmaterial, 1976) + Inlets (4 Spieler, 1977)

- Etudes Boreales I-IV (Cello, 1978) + Etudes Boreales I-IV (Klavier, 1978)

- Apartment House (großes Ensemble, 1976) + Renga (78 Stimmen, (1975/76)

-108 (Orchester, 1991) + One 8 (Cello, 1991) = 109a (Cellokonzert)

- 108 (Orchester, 1991) + One $e^{9}($ Shō, 1991 $)=109 b$

- 108 (Orchester, 1991) $+T w o^{3}$ (Shō und Conch Shells $)=110$ (Doppelkonzert)

\subsubsection{Werkverhältnisse: Beziehung statt Form}

„Die Beziehung von Dingen, die / gleichzeitig geschehen, ist spontan / und nicht unterdrückbar.“39

(John Cage)

In Bezug auf die Simultanaufführungen seiner Werke probierte Cage aus, ersetzte das eine durch das andere, zweifelte daran und ließ sich abermals überzeugen. An mehreren Stellen beschreibt er seine künstlerische Haltung als eine immerwährende Suche:

„Ich kann mir zum Beispiel vorstellen, daß Rozart Mix nicht zur gleichen Zeit gespielt wird wie die Song Books, ich meine mit den Sängern. Ich kann mir vorstellen, es durch meine Cartridge Music zu ersetzen. Ich möchte nur wissen, ob es ebenso lebendig wäre. Ich weiß es nicht. Während ich die Song Books schrieb, kam mir der Gedanke, daß es ein Fehler wär, die Song Books mit Rozart Mix oder

39 Cage, 45' für einen Sprecher, in: ders.: Silence, 2017, S. 83 . 
den Schlagzeugteilen von Atlas Eclipticalis oder sogar mit beidem zu überlagern. Als ich dann aber die von Davorin Jagodic und den Studenten aus Vincennes hergestellten Bänder hörte, insbesondere die Aufnahme von den Proben, bei denen wir uns alle im Theater befanden und von den Stimmen umgeben waren, da war ich wirklich glücklich.“40

„Auf jeden Fall werden wir in dem kommenden Konzert in New York die Song Books ohne das Concert und ohne Rozart Mix spielen. Und das wird zu einer ungewöhnlichen Situation beitragen, weil die meisten Solos in den Song Books aus einfachen Linienführungen ohne jegliche Begleitung bestehen. Demnach werden Umweltgeräusche in die Musik eindringen können. Sie werden das Mix herstellen. ${ }^{41}$

Die beiden Zitate verdeutlichen, wie selbstverständlich Cage die Möglichkeiten der Simultanaufführung in seine Praxis integrierte. So selbstverständlich, dass der Gedanke an Einzelaufführungen der ursprünglichen Werke für ihn auch eine Herausforderung darstellen konnte. Das könnte einerseits Ausdruck eines ehrlichen Zweifels oder Unvertrauens in die eigenen Werke sein, deren mögliche Schwächen sich so unverhüllt zeigen würden. Es zeigt aber viel mehr noch den Respekt vor der verborgenen Macht der spontanen Situativität des Ortes mit seiner individuellen Atmosphäre, dessen Stimmung von der Handlungsweise und der Haltung und Einstellung der ausführenden Musiker und des anwesenden Publikums geprägt wird. Im Vordergrund der Rezeption steht folglich die Beziehung zwischen den verschiedenen anwesenden Komponenten. Ihr unvorhersehbares Zusammenspiel kann eine Aufführung in eine aleatorische Polyphonie zwischen Werk und Umgebung verwandeln, kann vermeintliche Schwächen des Werks oder des Orts ebenso offenlegen wie verdecken.

Die Spontaneität dieser Beziehung entspricht dabei auch der Beziehung, die sich Cage für die Töne und Geräusche innerhalb seiner Werke vorstellt. In einem Brief an Boulez aus dem Jahre 1951 heißt es, „[... ] daß jedes Ding es selbst ist, daß seine Verhältnisse mit anderen Dingen auf natürliche Weise entstehen und nicht durch irgendeine Abstraktion des ,Künstlers' geschaffen werden $[\ldots]^{\star 42}$. Dieser Gedanke ist auch auf das Werkverhältnis in einer Simultanaufführung übertragbar. Indem die Form der Beziehung der Werke untereinander nicht durch den Komponisten schriftlich fixiert und damit eindeutig vorgegeben wird, existiert sie vor allem als eine von den Interpreten zu

40 Cage: Für die Vögel, S. 61.

41 Ebd., S. 16o. (Cage weist in der Fußnote darauf hin, dass diese Aufführung nicht stattfand).

42 Jean-Jacques Nattiez (Hg.): Dear Pierre-Cher John. Pierre Boulez und John Cage-der Briefwechsel, Hamburg: Europäische Verlagsanstalt, 1997, S. 108. 
nutzende unbestimmte Chance. Sie entspricht dem Anliegen einer Befreiung des Werks aus einem objekthaften und identitätsstiftendem Denken, sucht eine strukturelle Übereinstimmung mit der ihn umgebenden Welt, die sich nach Cage nicht als Objekt, sondern als ein Prozess präsentiert. ${ }^{43}$

Ebenso wie das Aufeinandertreffen der (akustischen) Ereignisse aus den spontanen Koinzidenzen jenseits einer kausalen Beziehung entsteht, so ist auch das von Cage im Kompositionsprozess immer wieder befragte I Ging in seiner Methodik auf dem „Prinzip des akausalen oder Synchronizitätszusammenhanges gegründet ${ }^{44}$ :

„Kausalität ist die Art, wie wir uns die Brücke zwischen zwei aufeinanderfolgenden Ereignissen vorstellen. Synchronizität aber bezeichnet den zeitlichen und sinngemäßen Parallelismus von psychischen und psychophysischen Ereignissen, welche unsere bisherige Erkenntnis nicht auf ein gemeinsames Prinzip reduzieren konnte. [...] Die Parallelereignisse lassen nämlich im Prinzip keinen gegenseitigen Kausalzusammenhang erkennen, weshalb sie eben einen Zufallscharakter tragen." 45

Cage, dessen I-Ging-Ausgabe von C. G. Jung herausgegeben worden war, vertrat die Auffassung, dass zwischen allen Klängen und Ereignissen bereits Beziehungen existieren. Eine beziehungs- und zusammenhaltstiftende Form, die ihre einzelnen Teile für das Verständnis des Ganzen funktionalisiert und damit kausale Brücken schafft, wäre in der Konsequenz überflüssig. Cage begreift das passive Geschehenlassen von Beziehungen zwischen gleichzeitig ablaufenden Dingen als natürlichen spontanen Prozess, der nicht künstlich gestört oder gehemmt werden sollte.

Die simultane Akkumulation verschiedener Werkräume bedeutet zusätzlich einen noch höheren Grad an Unbestimmtheit. Im Gespräch mit Daniel Charles stellt Cage einen Vergleich zu offenen Kompositionstechniken an, deren Steuerungsmechanismen die Elemente lediglich in einer sukzessiven Aufeinanderfolge zufällig verteilen. Für Cage stellt sich in diesem Fall die Indeterminiertheit als eine "mehr oder weniger perfektionierte Variante der Determiniertheit ${ }^{46}$ dar, und er differenziert zwischen verschiedenen Richtungen oder Graden von Indeterminiertheit: Einer linear ausgerichteten Indeterminiertheit, die den Raum der kompositorischen Vorstellungskraft zwar öffnet, aber sich immer noch auf den Radius des Vorstellbaren

43 Vgl. Cage: Für die Vögel, S. 89.

44 Carl Gustav Jung: Über Synchronizität, Reprint, Zürich: Buchclub Ex Libris, 1972, S. 40.

45 Ebd., S. 112.

46 Cage: Für die Vögel, S. 25 o. 
zurückführen lässt, wird der „Sprung in die Nicht-Linearität“47 als Ausdruck des Unbestimmten gegenübergestellt. Demnach würde die Simultaneität, die für Cage vor allem ein Bruch mit der Linearität ist, den erwünschten Bruch mit der Determiniertheit bedeuten, sofern sich das klangliche Resultat außerhalb des imaginierbaren und damit auch des intendierbaren Raumes des Komponisten befände. Die erweiterte Hörerfahrung kann also ebenso als erweiterte Imaginationserfahrung interpretiert werden. Denn jegliche Hörerfahrung, die sich außerhalb der genuinen Vorstellungskraft und Intention befindet, erweitert diese als „lebendige Hörerfahrung“ unweigerlich. Die Zuordnung der Determiniertheit zur linear geprägten Imagination und der Indeterminiertheit zur simultanen Erfahrung ist allerdings anzuzweifeln. Warum sollte eine simultane Schichtung prinzipiell weniger determinierbar sein als ein rein linearer Verlauf? Denn auch dieses ist letztlich eine Frage der mit der Zeit erworbenen Hör- und Imaginationserfahrung. Dem Einwand von Daniel Charles, dass auch das Ganze letztlich als Einheit und als linearer Verlauf wahrgenommen werden könne, entgegnet Cage, dass diese Einheit "nicht die Einheit einer starren fertigen Figur, sondern die einer vibrierenden ,NichtFigur'48 sei.

Die Durchdringung der verschiedenen Werkebenen führt zu einer Vielgestaltigkeit in der Werkausführung und zu einer konsequenten Verhinderung der Identitätsbildung des einzelnen Werks. Es entstehen immer neue Konstellationen: in Form der einzelnen Werkgestalt, in der Gleichzeitigkeit verschiedener Werkgestalten und in der Gleichzeitigkeit der Werkgestalten und ihrer akustischen Umgebung. An die Stelle des objekthaften Werkbegriffs wie er sich in der europäischen Kunstmusik ab 1800 zuzuspitzen begann - tritt bei Cage die Konstellation verschiedenster Klangereignisse, die wandelbar und permanent im Werden sind, ohne an einem Punkt zu einem greifbaren Objekt zu erstarren. Cage vergleicht sie in den Gesprächen mit Daniel Charles sinnfällig mit dem Sternenhimmel:

„Was die Konstellation in ein Objekt verwandelt, ist die Beziehung, die ich ihren Komponenten auferlege. Aber ich kann davon absehen, diese Beziehung zu postulieren, ich kann die Sterne als getrennt, jedoch eng zusammenstehend betrachten, fast zu einer einzelnen Konstellation verbunden. Dann habe ich einfach eine Sternengruppe. ${ }^{49}$

47 Ebd., S. 251.

48 Ebd.

49 Ebd., S. 87. 
Ein fixes Sternbild wie den Großen Wagen entstehen zu lassen wäre nach Cage bereits zu objekthaft gedacht.

\subsubsection{Die Aufführung als Rahmenöffnung des Werks}

Das Werk wird von Cage auch in seiner Einzelaufführung nicht als abgeschlossenes Objekt aufgefasst, sondern als eine offene Konstellation, die in eine Beziehung zu anderen akustischen Ereignissen treten kann, die zufällig gleichzeitig stattfinden. Ob eine gegenseitige Durchdringung stattfindet oder nicht, ist nicht primär eine Frage der Komposition, sondern der performativen und rezeptiven Strategie sowie des zufallsabhängigen Zusammenwirkens aller drei Komponenten.

Das Zusammenspiel in den Number Pieces ist ein anderes als im Concert for Piano and Orchestra. In den Number Pieces sollen die Mitwirkenden aufeinander hören, im Concert soll es keine Probe geben..$^{50}$ Inwieweit sich die Musiker über die Musik tatsächlich miteinander verbinden oder im Nebeneinander verharren, bleibt offen. ${ }^{51}$ Ebenso müssen die Hörer offen dafür sein, die Verbunden- oder Unverbundenheit koexistierender Klangereignisse und Strukturen als gleichwertige und vor allem als lebendige Erfahrung anzunehmen. Ähnlich wie im postdramatischen Theater rückt der ursprünglich komponierte Text in den Hintergrund. Die Aufführung ist nicht primär eine Repräsentation des Textes, sondern ein Akt der Emanzipation von diesem. In diesem Sinn ist das Werk nicht reproduzierbar.

Wie bereits dargelegt, zielte die Kritik von Cage am objekthaft geschlossenen Werkbegriff vor allem auf die in seinen Augen künstliche Trennung zwischen Kunst und Leben. Cage sah seine Werke als wesentlich offen und empfänglich für sämtliche Umgebungsgeräusche an, die nicht künstlich ausgeblendet, sondern zugelassen und als gleichwertig wahrgenommen werden sollten. Eine künstliche Schließung des Werks nach außen, eine deutliche Abgrenzung von der Welt ist nicht gewollt. Im Gegenteil, das Werk öffnet sich für die äußeren, zufällig stattfindenden Ereignisse in der Umwelt. Dieses unmittelbare Eindringen der Umwelt ins Werk oder auch umgekehrt, des Werkes in

\footnotetext{
50 Vgl. hierzu Metzger: Die freigelassene Musik, S. 196.

51 Siehe auch Martin Iddon: John Cage and David Tudor. Correspondence on Interpretation and Performance, Cambridge: Cambridge University Press, 2013, S. 173. Iddon verweist auf die alternativen Beziehungsformen zwischen den Interpreten auf der Bühne, die nicht auf das traditionelle Ensemblespiel gerichtet sind: „[...] the relationship between Tudor's and Cage's approaches was broadly similar to the relationship between Cage's music and Cunningham's choreography, in that both would occur at the same time and in the same venue, but there would be no attempt to coordinate the activities of the two, as in traditional choreographic approaches."
} 
seine Umwelt, bedeutet die Aufhebung einer Rahmensetzung, die gewöhnlich immer auch eine stabilisierende Funktion für ein Werk hat. Luhmann spricht in Hinblick auf das Kunstwerk von einer „,doppelte[n] Schließung “'52. Die innere Schließung bezieht sich in dem Sinn auf die Werkidentität, dass „jede Formsetzung einschränkt, was an weiteren Möglichkeiten übrig bleibt“53. Die innere Schließung ist der Werkseite immanent. Sie ist qualitativer und formaler Natur, indem durch die Formbildung eine Grenze erzeugt wird, die in "Formenunterschieden" ${ }^{54}$ wahrnehmbar wird und gleichermaßen "Identität und Differenz" ${ }^{\text {“5 }}$ garantiert. Sie basiert auf dem Ausschluss von Möglichkeiten. „Ein Kunstwerk, das sich im Unterschied zu allem anderen als Kunstwerk behauptet, schließt zunächst also alles andere aus und teilt die Welt ein in sich selbst und den übrig bleibenden unmarked space. “56 Neben der Schließung nach innen gibt es die Schließung nach außen. Sie bezieht sich auf die räumlichen, architektonischen und institutionellen Rahmenbedingungen der Aufführung mitsamt den üblichen Konzertabläufen und Ritualen, die das Aufführungsereignis rahmen, es vom Leben abgrenzen und dadurch ,jenen Transitraum [erzeugen], in [dem] „Kunsterlebnis und (eigenes) Leben die Führerschaft wechseln“57. Ziel ist, eine bestimmte Spannung, Sensibilität und Erwartungshaltung zu erzeugen, um die Aufmerksamkeit und Konzentration für eine bestimmte Dauer auf das akustische Werk richten zu können. Beide „Grenzlinien“, die innere wie die äußere, werden in den Simultankonstellationen bei Cage überschritten und geöffnet. In einem Happening im öffentlichen Raum fällt die äußere Schließung, die Grenzziehung zwischen dem Werk und der (Um-)Welt weg. Im Concert for Piano and Orchestra dagegen gibt es unzählige verschiedene Ausführungsmöglichkeiten und damit eine Flexibilität bezüglich des inneren Abschlusses. Diese ist auch in einem Werk wie 4'33 gegeben. Zusätzlich treten hier die äußeren Rahmenbedingungen einer Aufführung, ebenso wie die Außenklänge eines Werks durch das „fehlende, innere“ leere Werk noch stärker ins Bewusstsein. Ähnlich wie bei Bildarbeiten von Ben Vautier (art) oder Imi Knoebel (Keilrahmen, 1968) die aus leeren an der Wand hängenden Rahmen bestehen, mutiert der schließende Rahmen zum Gegenstand der Kunsterfahrung. Die Rahmenbedingungen, die es für die Kunsterfahrung eines Werkes braucht, treten durch die Leere des Werks umso

\footnotetext{
$5^{2} \quad$ Niklas Luhmann: Die Kunst der Gesellschaft, Frankfurt am Main: Suhrkamp, 1995, S. 53.

53 Ebd.

54 Ebd., S. 79.

55 Ebd., S. 83 .

56 Ebd., S. 61.

57 Mirjam Schaub: „Larger than life?!“, in: Uwe Wirth (Hg.): Rahmenbrüche Rahmenwechsel, Berlin: Kadmos, 2013, S. 179-197, hier: S. 179.
} 
stärker hervor und lassen die Korrelation zwischen den Innen- und Außenereignissen des Werks in den Vordergrund rücken. ${ }^{58}$

Aus dieser Perspektive scheint es ein naheliegender Schritt zu sein, ein musikalisches Werk nicht nur als implizit durchlässig für die Außenklänge, sondern auch als explizit durchlässig für das akustische Eigenleben eines anderen Werks zu betrachten. Die offene Form wird zum offenen Werk. Die zeitlichen, räumlichen und qualitativen Rahmensetzungen zwischen den Werken werden flexibilisiert und füreinander geöffnet, die Werk/Umweltverhältnisse verschoben. Die innere formale, identitätsstiftende und abgrenzende Rahmenlinie wird durchlässig für das Eigenleben eines anderen Stücks und mutiert zum Bindeglied zwischen dem Hier und dem Dort. Dagegen schafft eine Werkdifferenzierung nach innen neue Grenzen innerhalb des Werks und erzeugt neue Werk-Umwelt-Verhältnisse innerhalb einer gedachten Einheit. ${ }^{59}$

\subsection{Die Dialektik von Form und Offenheit bei Umberto Eco}

Während sich kaum ein Komponist von Poly-Werken, den ich befragt habe, auf John Cage berufen hat, gibt es zwischen Umberto Ecos Konzept der Offenheit, das er in seiner Schrift Das offene Kunstwerk ${ }^{60}$ entfaltet, und der Produktionsästhetik von Poly-Werken interessanterweise auffällige Schnittmengen. Das bedeutet nicht, dass Ecos Theorie die Komponisten bewusst geleitet hätte. Das wäre sehr unwahrscheinlich und wurde auch von keinem der Befragten thematisiert. Wahrscheinlicher ist es, dass - möglicherweise auch

$5^{8}$ Siehe auch: Anne Gilbert: „Leere Rahmen. Das Unsichtbare des Sichtbaren sichtbar machen“, in: Wirth (Hg.): Rahmenbrüche Rahmenwechsel, S. 217-237. Hier wird auch auf die Tradition leerer Ausstellungen hingewiesen, die die institutionelle Rahmung in der bildenden Kunst thematisieren. Beispiele sind die leere Ausstellung Le Vide von Yves Klein in einer Pariser Galerie 1958 oder auch die Ausstellung Von da an/A partir de là aus dem Jahre 1975 von Daniel Buren, der alle Kunstwerke aus dem Museum Mönchengladbach entfernen ließ. Auch die Berner Kunsthalle widmete sich 2009 einer großen Retrospektive leerer Ausstellungen.

59 Siehe dazu Armin Nassehi, Die Zeit der Gesellschaft. Auf dem Weg zu einer soziologischen Theorie der Zeit, Wiesbaden: VS Verlag für Sozialwissenschaften 2008 (1993), S. 238-245. Seine Ausführungen zum Zusammenhang zwischen Zeit und Gesellschaftsdifferenzierung im Kapitel „Systemdifferenzierung und Gleichzeitigkeit“ (S. 238-234) lassen sich für diesen Aspekt zum Teil auf simultane Werkkonstellationen übertragen: „So differenziert sich ein System in Teilsysteme aus, die füreinander Umwelt sind, wobei es sich bei dieser Umwelt um eine, innergesellschaftliche Umwelt handelt. [...] Ihre Teilsysteme dagegen erleben sich in einer kommunikativen Umwelt - und sind doch Systeme mit operativ geschlossener Autopoiesis." (S. 240 ff.)

6o Eco, Das offene Kunstwerk. 
in Abgrenzung zu Cage - eine bewusste oder auch unbewusste ästhetische Gegenposition eingenommen wurde, die auch noch mehrere Jahrzehnte nach Erscheinen von Ecos Schrift eine künstlerische Relevanz hat.

In groben Zügen zusammengefasst, unterscheidet Eco in seiner Theorie verschiedene „Intensitätsebenen “ von Offenheit ${ }^{61}$, die er graduell voneinander abgrenzt: Die Offenheit ersten Grades ist allen Kunstwerken immanent, denn auf einer rezeptiven Ebene ist jedes Kunstobjekt insofern offen, als es in seinem Sein immer auf eine theoretisch unendliche Vielzahl subjektiver Perspektiven trifft, die es erst vollenden würden. ${ }^{62}$ Eco spricht von einer "perzeptive $[n]$ Ambiguität" ${ }^{\prime 63}$ und bezieht diese Beobachtung vor allem auf den Akt der Rezeption durch den Empfänger der künstlerischen Botschaft. Die Offenheit zweiten Grades entwickelt diesen Gedanken weiter und formt ihn zum künstlerischen Programm in dem Sinne, dass es sich zwar um abgeschlossene Kunstwerke handelt, die „aber dennoch ,offen' sind für ständige Neuknüpfungen von inneren Beziehungen, die der Rezipierende im Akt der Perzeption der Reiztotalität entdecken und auswählen soll“64. Diese Ästhetik findet ihren konkreten Ausdruck in den sogenannten „Kunstwerke[n] in Bewegung“65, die den Gedanken der inneren Beziehungsvielfalt auf die innerformale Organisation eines Werks ausweiten und damit eine dritte Ebene der Offenheit verkörpern. Bereits auf der Stufe der Komposition wird nun statt eines einzigen festgelegten Verlaufs ein Möglichkeitsfeld geschaffen, das den ausführenden Musikern innerhalb eines vom Komponisten festgelegten Rahmens verschiedene Optionen bietet. Die Gestalt eines Werkes wird in der Aufführungssituation geformt. Damit wird die Zahl interpretativer Möglichkeiten nochmals gesteigert. Offenheit wird nun auch konzeptuell in das Werkobjekt integriert. ${ }^{66}$ Dieses Vorgehen richtet sich gegen Wiederholbarkeit, Gewohnheit und Eindeutigkeit zugunsten von Austauschbarkeit, Permutabilität und Mehrdeutigkeit. ${ }^{67}$ An die Stelle der Einsinnigkeit und des Absoluten tritt das

\footnotetext{
61 Ebd., S. 57 und S. 138 f.

62 Ebd., S. 3 .

63 Ebd., S. 50.

64 Ebd., S. 57.

65 Ebd., S. 42.

66 Das musikalische Werkobjekt durchläuft folglich zwei Interpretationsphasen. Eco versteht unter einem Interpretationsprozess sowohl das "passive“ Rezipieren als auch das „aktive“ Interpretieren. Er bezeichnet sie als „unterschiedliche Erscheinungen ein und derselben interpretativen Einstellung“" (Eco, Das offene Kunstwerk, S. 29).

67 Als Beispiele führt Eco hier die Mobiles von Alexander Calder, Kompositionen von Boulez, Stockhausen, Berio und Pousseur sowie Stéphane Mallarmés Livre an, ebenso Beispiele aus dem Industriedesign wie umbaubares und multifunktionelles Mobiliar und im Falle der Architektur die verschiebbaren Wände eines Gebäudes (ebd., S. 42 f.).
} 
Optionale, Relationale und Mehrfunktionale, das sich nicht mehr eindeutig bestimmen lässt. Eco schreibt das Streben nach einer programmatischen Offenheit den "modernen Poetiken“ zu.

Die Ergänzung der Werkversionen und die Unmöglichkeit, das Werk in seiner Gesamtheit gleichzeitig zu erfassen, entspricht dem Komplementaritätsmodell, das Eco nach naturwissenschaftlichen Beschreibungen Werner Heisenbergs und Nils Bohrs auf das Konzept offener Kunstwerke übertragen hat:68

„Von Mallarmés Livre bis hin zu den musikalischen Kompositionen, die wir untersucht haben, bemerken wir die Tendenz, das Kunstwerk so zu organisieren, daß keine Ausführung des Werkes mit einer letzten Definition von ihm zusammenfällt; jede Ausführung erläutert, aber erschöpft es nicht, jede Ausführung realisiert das Werk, aber alle zusammen sind komplementär zueinander, jede Ausführung schließlich gibt uns das Werk ganz und befriedigend und gleichzeitig unvollständig, weil sie uns nicht die Gesamtheit der Formen gibt, die das Werk annehmen könnte." ${ }^{\prime 69}$

Der Rezipient eines offenen Kunstwerkes nimmt nur einen Ausschnitt der Möglichkeiten wahr: Die „unvollkommene Kenntnis eines Systems [ist] essentielle Komponente seiner Formulierung.“70 Eco verweist auf die Beziehung zu Aussagen, die in Bezug auf wissenschaftliche Erkenntnisse gemacht wurden, und zitiert Niels Bohrs Erkenntnis:

„Demzufolge kann das unter verschiedenen Versuchsbedingungen gewonnene Material nicht mit einem einzelnen Bilde erfaßt werden; es ist vielmehr als komplementär in dem Sinne zu betrachten, daß erst die Gesamtheit aller Phänomene die möglichen Aufschlüsse über die Objekte erschöpfend wiedergibt."71

\subsection{Die Offenheitskonzepte von Cage und Eco im Vergleich}

Der Begriff „offene Form“ in der Neuen Musik bezieht sich zunächst auf einen Paradigmenwechsel in der formalen Ästhetik, indem er das Beziehungsgeflecht sowohl quantitativ als auch qualitativ erweitert. Ein undifferenzierter

\footnotetext{
68 Eco, S. 49.

69 Ebd.

70 Ebd.

71 Niels Bohr: „Diskussion mit Einstein über erkenntnistheoretische Probleme in der Atomphysik“, in: Albert Einstein als Philosoph und Naturforscher, hg. von Paul Arthur Schilpp, Stuttgart: Kohlhammer, 1955, S. 115-15o, hier: S. 123.
} 
Gebrauch birgt die Gefahr eines unkritischen Umgangs mit dem Begriff wie die bereits angesprochene oberflächliche Ineinssetzung der Kategorien Form und Werk (vgl. Kapitel 1.2). Statt um die eine „offene Form“ handelt es sich vielmehr um verschiedene „Formen von Offenheit" - von denen auch der Werkgedanke betroffen sein kann und denen durchaus unterschiedliche Weltanschauungen zugrunde liegen können. In der Musik ist das Konzept der Offenheit als ästhetisches Programm im Verlauf des 20. Jahrhunderts effektiv auf drei verschiedenen Beziehungsebenen wirksam geworden:

1. in der Auflösung der eindeutig fixierten innerformalen Beziehungen und Verhältnisse eines Werks;

2. in der Auflösung eines eindeutigen Abgrenzungsverhältnisses zwischen Werk und kontextualisierender Umgebung und damit der Etablierung einer extra- oder interformalen Ebene, die sich mit der innerformalen Ebene verschränken kann;

3. in der Aufwertung der interpretativen Praxis.

Sowohl die Auflösung der eindeutig bestimmten innerformalen Beziehungen des "Werks" als auch die Auflösung der konventionellen extraformalen Beziehungen zur "Welt" brachten systematisch durchlässigere und flexiblere Relationsverhältnisse hervor. Für die werkinnere Formgestaltung konnten dies beispielsweise Austauschbarkeit, Verschiebbarkeit, freie Wahl formaler Abschnitte oder parametrischer Setzungen und Einbettung der Improvisation sein. Form wurde zunehmend zu einer Art Bausatz, aus dem die Interpreten die vorgegebenen Bausteine individuell zusammensetzen konnten.

Mit der Aufhebung einer eindeutigen Abgrenzung zwischen „Werk und Welt" weitete sich der Aktionsradius musikalischer Aufführungen aus. Bereits die Auflösung strenger frontaler Sitzordnungen im Konzertsaal, der Einbezug des Publikums, die Verlagerung des Konzertereignisses in den öffentlichen Raum oder die Integration von Umweltgeräuschen können einfache Mittel sein, um bestehende Ränder und Grenzen zu verwischen, da sie immer auf eine Veräußerlichung der Interaktionen zwischen den Teilnehmenden gerichtet sind und versuchen, die Kontextualisierung des Werkes bewusst zu gestalten. Diese verschiedenen Wahl- und Interaktionsmodi weiteten den Interpretationsradius der Musiker sowohl quantitativ als auch qualitativ aus und vergrößerten ihre Handlungsräume. Einerseits kamen neue Interpretationsebenen hinzu, auf denen der Interpret Entscheidungen und Individualisierungen vornehmen konnte, sei es die formale Anordnung von Abschnitten oder die individuell imperfekte Ausführung komplexer Partituren. Andererseits wurde ein erweitertes Engagement vorausgesetzt, das über den reinen und "schönen“ Vortrag hinausging und schließlich in die Performancekunst mündete. Nicht alle dieser Handlungsräume waren neu. 
Manche bildeten bereits einen selbstverständlichen Teil der Kompositionsund Interpretationspraktiken früherer Jahrhunderte. Im Zusammenhang des 20. Jahrhunderts erweiterten sie durch ihre Programmatik nicht nur die Werkbildung, sondern begründeten auch eine Reihe neuer Genres wie Konzept-, Klang-, Installations- oder Performancekunst.

Wie kein anderer Komponist vor und nach ihm hat Cage seine Stücke auch in simultanen Fassungen im Sinn gehabt. Schon aus diesem Grund ist sein Ansatz für das Thema relevant und voraussetzungsreich. Dennoch sind seine Werke auf eine ganz andere Art und Weise Ausdruck eines offenen Denkens und Vorgehens, als es im Poly-Werk der Fall ist. Dieses kann - im Gegensatz zu den Werken Cages - vor dem Hintergrund der Theoriebildung von Umberto Eco diskutiert werden. Um die unterschiedlichen Bedeutungsebenen des Offenen herauszuarbeiten und eine konstruktive formästhetische Verortung des Poly-Werks und der Simultanaufführung bei Cage vorzunehmen, lohnt sich ein direkter und überblicksartiger Vergleich zwischen den beiden unterschiedlichen Ansätzen.

Ecos Darstellung des „Offenen“ steht in vielen Punkten im Widerspruch zu den Aussagen, die Cage in seinen Schriften und Interviews gemacht hat. Cage ist politisch, bezieht die soziale Dimension mit ein, argumentiert religiös und beruft sich auf fernöstliches Denken und den Zen-Buddhismus. Die Theoriebildung von Umberto Eco stützt sich auf Beobachtungen, Analysen und Analogien. Sie spürt den kulturellen und historischen Bedingungen und dem Weltbild nach, die den offenen Formen in der Kunst zugrunde liegen. Um die Entwicklung der Kunst der Moderne nachzuvollziehen, ist sein Blick auch in die Vergangenheit gerichtet. Eco argumentiert auf der Basis naturwissenschaftlicher und informationstheoretischer Beschreibungsweisen und Analogien und mithilfe von Beispielen aus der modernen Physik, der Quantenphysik, der Metaphysik, der Kommunikationstheorie und der Semiotik. Eine vergleichende Reflexion beider Auffassungen und ihre Übertragung auf die kompositionstheoretischen Konzeptionsmöglichkeiten ist aufschlussreich für die Aufdeckung der zugrunde liegenden Weltbezüge. Zudem bestätigt sie, dass sich die Auflösung einer eindeutigen Werkvorstellung in der Mitte des vergangenen Jahrhunderts mitnichten einseitig als Negation des Werkbegriffs auffassen lässt. Sie führte vor allem zu einer Auffächerung der Möglichkeiten, den Werkbegriff weiterzuentwickeln.

Wie bereits mehrfach gesagt, besteht ein Hauptunterschied zwischen einer Simultanaufführung und einem Poly-Werk darin, dass die Simultanaufführung ein rein interpretativer und die Simultaneisierung im Poly-Werk ein kompositorischer Akt ist. Im ersten Fall müssen die Interpreten mithilfe ihrer Analysefähigkeit, ihres Wissens, ihrer Intuition, Fantasie und Erfahrung selbst 
herausfinden, welche Stücke sich mit anderen kombinieren lassen. Sie müssen Entscheidungen über die passenden Tempi treffen und Dauern und Ereignisverläufe der Stücke berücksichtigen, um zu einer neuen Form zu kommen. Die Simultaneisierung ist hier eindeutig Teil der Interpretation. Bei Cage sind die Simultanaufführungen zwar kompositorisch intendiert, jedoch gibt es keine eindeutigen Angaben zur Zusammenführung. Interpretation hat hier den Status einer sich von der Komposition emanzipierenden Praxis. Als solche vermag sie die ursprünglichen Intentionen eines Werks zu unterlaufen.

Es ist ein allgemeines Merkmal offener Kunstkonzepte, dass die interpretative Praxis eine Aufwertung gegenüber der Produktion erfährt und der Interpret und der Hörer sich vom Komponisten und seinem Werk emanzipieren. Jedoch kann diese Aufwertung mit unterschiedlich viel Spielraum für die Interpreten verbunden sein und dadurch unterschiedliche Konsequenzen auf das Verhältnis Komposition - Interpretation - Rezeption haben. Cage stellt die Getrenntheit der Elemente dieser dreistufigen klassischen Konstellation infrage..$^{72}$ Andererseits negiert er ihren natürlichen zielorientierten und linearen Zusammenhang in Bezug auf die musikalische Werkerfahrung.

„Ich habe einmal gesagt, Musik zu schreiben sei etwas anderes als Musik aufzuführen oder sich anzuhören. Das sind drei vollkommen verschiedene Dinge. Was man hört, was aus dem Geschriebenen entsteht, unterscheidet sich vollkommen von der Tatsache, daß ich es schreiben konnte."73

Dieser vermeintliche Widerspruch löst sich dann auf, wenn man Cages Kritik in beiden Fällen als Kritik an einer festgesetzten Hierarchie versteht. Um Hierarchiebildung zu umgehen, muss Beziehungslosigkeit hergestellt werden. Durch ein „zielloses Schreiben“, das er als „reines Schreiben“ anerkennt, würde auch eine „reine Aufführung“ und ein „reines Hören“ möglich, das jene Beziehungslosigkeit möglich macht. ${ }^{74}$

72 Siehe beispielsweise Cage im vierten Gespräch mit Daniel Charles in Cage: Für die Vögel, S. 153. Im Gespräch mit Richard Kostelanetz beschreibt Cage anhand einer stattgefundenen Aufführung an der University of Wisconsin, bei der er gemeinsam mit dem Publikum Klangrouten entwickelte und beging, einen möglichen Ansatz: „Aus diesem Grund versuche ich in meinen Aufführungen verstärkt auf eine Situation hinzuarbeiten, die den Unterschied zwischen Aufführenden und Publikum aufhebt. Dabei denke ich nicht an eine vom Komponisten einkalkulierte Publikumsbeteiligung. Vielmehr schwebt mir eine Musik vor, die sich aus den Aktivitäten der Interpreten und dem sogenannten Publikum ergibt." (Kostelanetz:John Cage im Gespräch, S. 101.)

73 Cage: Für die Vögel, S. 62.

74 Ebd., S. 62. 
Auch Eco geht von einer Interpretationsästhetik aus und wertet diese damit auf. Jedoch differenziert er zwischen diesen drei Stadien, indem er sie als drei perspektivisch unterschiedliche Ebenen des Kunstobjektes anerkennt. Das moderne Kunstwerk als Signifikant (Bedeutungsträger) enthält für ihn eine Mehrzahl von Signifikaten (Bedeutungen). Damit wird die Vielfalt der Deutungsmöglichkeiten bereits in den Werkgedanken integriert. An die Stelle einer „signifikanten Form“ tritt eine Form, die sich aus dem „Spiel der Signifikate ${ }^{\prime 75}$ ergibt. Diese Sicht entspricht auch dem Wesen des Poly-Werks. Auch hier integriert der Komponist mit den unterschiedlichen Kontextualisierungsmöglichkeiten von Anfang an mehrere Deutungsmöglichkeiten des musikalischen Textes und gibt diese an die Interpreten weiter. Sofern also das Verhältnis zwischen der schöpferischen und der interpretierenden Seite von Mehrdeutigkeit und Ambiguität geprägt ist, spiegelt Ecos „Modell des offenen Kunstwerks“ auch diese vielschichtige „Rezeptionsbeziehung “ wider $^{76}$ Darüber hinaus wird die interpretative Praxis im Poly-Werk bereits Teil der kompositorischen Praxis und verschränkt sich mit dieser, da der Komponist das Festgeschriebene durch die anderen Werke unterschiedlich kontextualisiert. Dadurch wird die Absolutheit des Werks und seiner Interpretation relativiert.

Aus Ecos Sicht ist das „Programm der Offenheit“ als Überbestimmung angelegt. Eine eindeutige Bestimmung wird dabei genauso vermieden wie Unbestimmtheit. Im Gegensatz dazu ist bei Cage Unbestimmheit oder auch indeterminacy das ästhetische Leitprogramm. Es wirkt hier als Möglichkeit der Auflösung von Bestimmung und Begrenzung. Um dies zu erreichen, strebt Cage nach Nichtintentionalität, die sich primär im Befolgen bestimmter äußerer Regeln, im Einbezug des Zufalls, im Befragen des I Ging etc. ausdrückt. Die Integration entsubjektivierter und damit objektivierter Strategien soll eine rezeptive Haltung, eine „entpersonalisierte[...] Komposition“"77 bewirken und die subjektive Intentionalität und Kontrolle des Komponisten über das Endresultat unterwandern. Auch simultane Verfahren wie ein "Musicircus“ oder eine konkrete Werküberlagerung sind bei ihm Ausdruck des Bedürfnisses, Ordnung, Linearität, Determination und die Intentionalität des Komponisten zu brechen (vgl. Kapitel 1.3.3). Dieser Gedanke markiert einen zentralen Gegensatz zu einer Produktionsästhetik die den absichtsvollen Willen und die Intentionen des Komponisten in den Mittelpunkt stellt. ${ }^{78}$ Aus Sicht von Eco

75 Vgl. hierzu Eco, Das offene Kunstwerk, S. 15.

76 Ebd., S. 15 .

77 Christoph Metzger: John Cage - abstract music. Zwölf Vorlesungen, Saarbrücken: Pfau, 2011, S. 31.

78 Für Martin Iddon markiert dieser Punkt den grundlegenden Unterschied zwischen der sogenannten „Darmstädter Schule“ und Cage: „The fundamental distinction, then, was not 
sollte der Komponist zwar außermusikalische Organisationsverfahren oder Zufallsprozesse integrieren, diese aber auch zu beherrschen lernen, um seine determinierende, bestimmende Kraft zu behalten. Damit ist er auf einer Linie mit Pierre Boulez, der vom „dirigierten Zufall“79 spricht. Der Komponist soll danach streben, Mehrdeutigkeit intentional zu gestalten, das Werk trotzdem zu einer organischen Einheit zu formen und die Kontrolle nicht vollständig abzugeben. Dabei wird der Dialektik von Information und Bedeutung oder von Qualität und Quantität auch wieder besondere Bedeutung geschenkt und sie als Problem diskutiert. Denn die Abkehr von der Eindeutigkeit hin zu einer mehrwertigen Ordnung bedeutet eben auch einen Zuwachs an Information mit einer Tendenz zum Rauschen und zur Unordnung. Eco unterscheidet zwischen

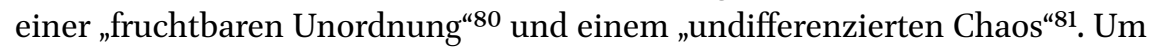
letzterem entgegenzuwirken, sei eine „beherrschte[...] Unordnung“ nötig, beispielsweise durch abgesteckte Möglichkeiten innerhalb eines abgegrenzten Rahmens und „Keime der Formhaftigkeit “82, die einem möglichen Chaos entgegenwirkten.

Eco grenzt das Programm der Offenheit nach zwei Seiten ab: Einmal gestaltpsychologisch von der "gute[n] Form“ mit der "geringste[n] Information“ und "größte[n] Redundanz“, die dem „,Zustand größter Wahrscheinlichkeit eines fluktuierenden Wahrnehmungsganzen “ 83 entspricht, sowie auf der anderen Seite von Willkür, Chaos, Beliebigkeit und Anarchie. Beide, Eindeutigkeit und Beliebigkeit (die keine Bedeutung hat), sind zwei Seiten einer Medaille, da sowohl Eindeutigkeit als auch Bedeutungslosigkeit das Aufkommen von Ambiguität verhindern. ${ }^{84}$

Die Prinzipien der Cage'schen Nichtintentionalität und Unbestimmtheit sind mit dem Poly-Werk nicht vereinbar. Ecos Argumentationslinie stimmt

between serial method and chance operations at all, but rather between Europeans, who wanted to be,composers' through the operations of their wills and compositional desires, and Americans, who were willing to allow music to occur unpurposively." (Martin Iddon: New Music at Darmstadt. Nono, Stockhausen, Cage, and Boulez, Cambridge: Cambridge University Press, 2013, S. 215.)

79 Boulez: Alea, S. 178.

8o Eco, Das offene Kunstwerk, S. 9 .

81 Ebd., S. 13 .

82 Ebd.

83 Ebd., S. 15 o.

84 Vgl. hierzu auch Thomas Bauer: Die Vereindeutigung der Welt. Über den Verlust an Mehrdeutigkeit und Vielfalt, Stuttgart: Reclam, 2018, S. 29 f. Bauer unterscheidet zwei Möglichkeiten, Ambiguität zu verhindern: 1. die Eindeutigkeit und 2. den völligen Mangel an Bedeutung, sodass „alle Interpretationen gleich gültig“ sind und damit die Sache insgesamt bedeutungslos ist. 
insofern mit dem Poly-Werk überein, als ein einziges Werk immer auch mehrere Funktionen übernimmt und damit bereits auf der kompositorischen Ebene zu einer Überbestimmung und Mehrdeutigkeit des musikalischen Textes gelangt. Die sich ergebende Dichte der Überlagerung führt auch zu einer höheren Informationsdichte, basiert aber immer auch auf einer willentlich geformten Ordnung, welche Zufallselemente höchstens gezielt einkalkuliert, aber nicht die Kontrolle über das Endresultat aus der Hand gibt. Daher verkörpert das Poly-Werk weder die einfache "gute“ und leicht voraussehbare Form, von der Eco die "offene Form“ abgrenzt, noch ist es auf der anderen Seite Ausdruck einer "anarchischen“ Form.

Cage sieht sich selbst als Neuerer und seinen künstlerischen Weg als Gegenpol zur europäischen Tradition, Kultur und Werkästhetik. Eco hingegen leitet das Programm der Offenheit aus einem Traditionsstrang der europäischen Kultur ab, der vor allem eine Entwicklungslinie freiheitlichen Denkens abbildet. Offenheit ist ein Projekt der Moderne, das sich erstmals im Barock als Reaktion auf die kopernikanische Wende im 16. und 17. Jahrhundert ausdrückt, „weil sich hier zum erstenmal der Mensch der Norm des Kanonischen [...] entzieht und in Kunst und Wissenschaft einer in Bewegung befindlichen Welt gegenübersteht, die ein schöpferisch-erfinderisches Verhalten von ihm verlangt" ${ }^{485}$.

Aus dieser Traditionslinie heraus betrachtet Eco Kunst weiterhin als Komplement der Gesellschaft, die sie durch eine „bestimmte Disposition ihrer Formen ${ }^{486} \mathrm{zu}$ charakterisieren vermag: „Der eigentliche Inhalt des Kunstwerks wird somit seine Art, die Welt zu sehen und zu beurteilen, ausgedrückt in einem Gestaltungsmodus [...] $]^{487}$. Als „epistemologische Metapher ${ }^{488}$ spiegelt die Kunst die Realität und auch die Fortschrittlichkeit künstlerischen Denkens wider: „[...] der Komponist ist fortschrittlich in dem Maße, wie er auf der Formebene eine neue Art, die Welt zu sehen, durchsetzt [...] $]^{489}$ und als „offene Struktur [...] die Ambiguität unseres In-der-Welt-Seins reproduziert ${ }^{490}$. Das Verhältnis von Werk und Welt ist ein komplementäres, eine Gegenüberstellung, wobei die Weltsicht sich nach Eco primär über die Formgebung eines Werks artikuliert. Dieser Ansatz steht völlig konträr zum typischen Neue-Musik-Diskurs, der Fortschrittlichkeit in erster Linie über das Material definiert hat und lediglich die

85 Eco, Das offene Kunstwerk, S. 35. Ebenso führt Eco an anderer Stelle allerdings auch Gedichte von Petrarca als Beispiele offener Kunstformen an (S. 124 f.).

86 Ebd., S. 268.

87 Ebd., S. 271.

88 Ebd., S. 46.

89 Ebd., S. 269.

$90 \quad$ Ebd., S. 286. 
Negation der Form - parallel zur Negation der Werkkategorie - als Ausdruck für Fortschrittlichkeit interpretierte. ${ }^{91}$ Da es in vielen Poly-Werken Anhaltspunkte für ein Formdenken gibt, das auch als Ausdruck einer mehrdeutigen Weltsicht auslegbar ist - beispielsweise die mehrfache und polyvalente Einbettung des Einzelnen in ein funktional differenziertes System -, ist das Merkmal einer auf Ambiguität und Mehrdeutigkeit ausgerichteten Formendisposition einer der interessantesten Schnittpunkte zwischen den Poly-Werken und Ecos Theorie.

Die von Cage artikulierte Sicht hebt die Distanz zwischen Werk und Welt auf. Bereits die Überlagerung eines Werks mit seiner zufälligen akustischen Umwelt kann Cage zufolge eine Simultanaufführung sein (vgl. Kapitel 1.3.3). Cage strebt nach einer Aufhebung der in seinen Augen künstlichen (und ebenso institutionellen) Trennung zwischen Kunst und Leben. Beide sollten ineinander übergehen. „Ich glaube, die moderne Kunst hat das Leben zur Kunst gemacht, und jetzt finde ich es an der Zeit, daß das Leben [...] alles in Kunst verwandelt $[\ldots]]^{492}$ Das „Werk“ als isoliertes Pendant zur Welt erfährt auch so wieder eine Auflösung.

Bei Eco wird entsprechend seiner Argumentationslinie diese Auflösung nicht vollzogen, sondern er zielt auf eine „Dialektik zwischen Form und Offen-

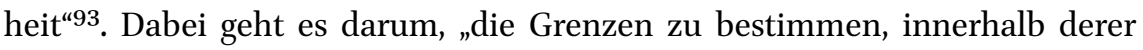
ein Kunstwerk die größte Mehrdeutigkeit verwirklichen und vom aktiven Eingriff des Konsumenten abhängen kann, ohne damit aufzuhören, Kunstwerk zu sein “94. Statt für die Negation spricht sich Eco für eine erweiterte und affirmative Auffassung der Werkästhetik aus und erkennt Form weiterhin als „Grundbedingung für die Kommunikation“95 an. Offenheit und Geschlossenheit stellen in seinem System keine einander ausschließenden Kategorien, keine Dichotomie dar. Stattdessen gibt es verschiedene Grade des Offenen in Bezug auf das Geschlossene, das weiterhin anerkannt wird. Auch damit entspricht sein Ansatz der Konzeption von Poly-Werken, denn bei ihnen erscheinen Geschlossenheit und Offenheit ebenfalls nicht als Dualität, sondern als gleichwertige und einander bedingende Formkategorien, die einander

$91 \quad$ Erst in den letzten Jahren sind einige Arbeiten erschienen, die diese Fehleinschätzung zu korrigieren versuchen oder alternative Sichtweisen und Zugänge zum Formbegriff vorschlagen. Siehe Holzer: Zur Kategorie der Form in neuer Musik, oder Cosima Linke: Konstellationen - Form in neuer Musik und ästhetische Erfahrung im Ausgang von Adorno. Eine musikphilosophische und analytische Untersuchung am Beispiel von Lachenmanns „Schreiben. Musik für Orchester“, Mainz: Schott, 2018. Kostelanetz: John Cage im Gespräch, S. 163 (nach Robin White/John Cage, Interview bei der Crown Point Press, View 1, Nr.1, Oakland, Point Publishers, 1978).

93 Eco, Das offene Kunstwerk, S. 129.

94 Ebd., S. 8.

95 Ebd., S. 182. 
nicht einfach negieren, sondern vor allem in ihrer Ausbalancierung präsent sind.

In einem Punkt stimmen Cage und Eco überein. Sowohl bei Cage als auch bei Eco geht die Öffnung des Werkkonzepts mit einer offenen geistigen Grundhaltung einher. Eco plädiert für einen „Menschentyp[...], der offen ist für eine ständige Erneuerung seiner Lebens- und Erkenntnisschemata, der produktiv an der Entwicklung seiner Fähigkeiten und der Erweiterung seiner Horizonte

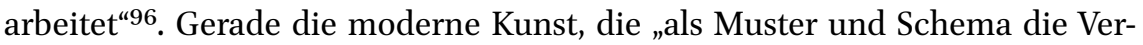
gänglichkeit der Modelle und Schemata erwählt ${ }^{497}$, wirke auf den heutigen Menschen stimulierend. Sie könne ihm in einer Welt, die permanent auf eine Übernahme von ",guten Formen “998 aus ist, „eine Möglichkeit zur Selbstfindung und Autonomie ${ }^{\prime 99}$ zeigen. Bei Cage artikuliert sich diese Haltung als Nichtintentionalität, die er auch als „response ability " ${ }^{100}$ bezeichnet. Es handelt sich um die Fähigkeit zu reagieren und empfänglich zu sein, durch Unbestimmtheit zu einer Offenheit und Neugier für Fremdes und Unbekanntes zu gelangen, die den eigenen Erfahrungshorizont übersteigt und für neue Erfahrungen bereit ist: „Ich glaube, wir müssen zu einer Erfahrung bereit sein, die nicht durch Verstehen, sondern durch Aufgeschlossenheit zustandekommt." ${ }^{\text {"101 }}$

Zusammenfassend lässt sich sagen, dass Offenheit und Geschlossenheit bei Cage als Dualismus auftreten, während Eco ähnlich wie Wölfflin (vgl. Kapitel 1.2) Offenheit als graduelle Modifikation des geschlossenen Werks ansieht. Bei ihm handelt es sich in erster Linie um eine innerformale Offenheit des geschlossenen Werks, da der Rahmen oder die "Werkgrenzen“ im traditionellen Sinn weiterbestehen. Dies entspricht auch der Anlage eines Poly-Werks: Aus der Perspektive der ganzen Werkentität handelt es sich um eine innerformale Flexibilität und Verschiebbarkeit, die sich allerdings hier auf konkrete gestaltete Werkstränge und nicht mehr nur auf einzelne Bausteine bezieht. Aus der Perspektive des einzelnen Werkstrangs sind die einzelnen Werke im Ganzen füreinander auch akustische Umgebungen, stellen eine Kontextualisierung dar, sodass es sich auch um interformale Beziehungen handelt, die auf die innerformalen zurückwirken können. Da diese allerdings immer den gleichen Wahrnehmungsmodus ansprechen und nie den medialen Rahmen des komponierten Werks verlassen, sind sie keine kontinuierliche

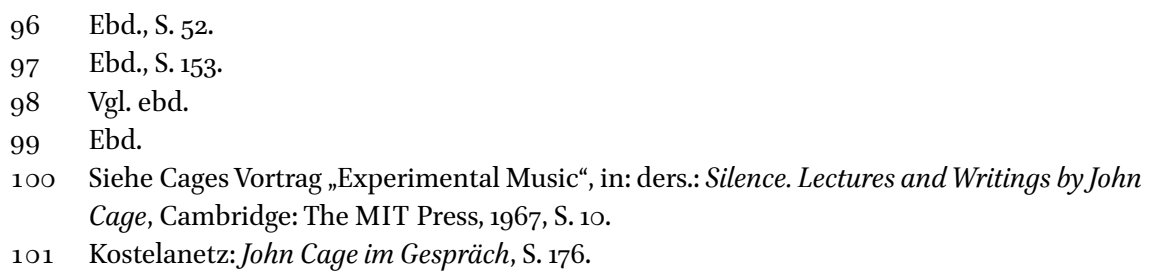


Öffnung zur Welt und zum Leben oder Fortsetzung derselben im Sinne von Cage. Das Poly-Werk ist weiterhin eindeutig von der Welt abgrenzbar, markiert eine Differenz, ist im Sinne von Ecos Theorie auch weiterhin ein Komplement der Welt, ein Spiegel zu ihr. Bei Cage wiederum greift diese innerformale Offenheit auch auf das Verhältnis zwischen Werk und Welt aus. Beides soll sich ineinander fortsetzen und ineinander übergehen.

Während der Begriff der Offenheit bei Cage sehr treffend mit Leere gleichgesetzt werden kann, die sowohl eine rezeptive Haltung als auch eine rezeptive Produktionsästhetik befördern soll, ${ }^{102}$ kann über Ecos offenen Formbegriff gesagt werden, dass er mehrere komplementäre Lösungen einschließt: „Offenheit und Dynamik eines Kunstwerks bestehen hingegen im Sich-verfügbarMachen für verschiedene Integrationen, konkrete produktive Ergänzungen, die es von vornherein in den Spielraum einer strukturellen Vitalität einfügen [...]." ${ }^{“ 103}$ Diese Aussage spricht in erster Linie eine aktive und dynamische Interpretationshaltung an. Vor allem das von Eco in die Diskussion eingeführte Komplementaritätsmodell, das besagt, dass keine einzelne Ausführung des Werks es in seiner Gesamtheit abzubilden vermag, schlägt eine weitere entscheidende Brücke zum Poly-Werk und stellt rückblickend die Möglichkeit bereit, simultane Integration, wie sie im Poly-Werk vollzogen wird, als auskomponierte Variante und Weiterentwicklung eines offenen, dynamischen und ambiguen Werkkonzepts im Sinne von Eco weiterzudenken.

Auch auf der interpretativen Ebene ist die Simultanaufführung näher bei Cage, das Poly-Werk dagegen näher bei Eco angesiedelt. Ist die Simultaneisierung bei Cage eindeutig eine rein interpretative Praxis, bleibt sie im Poly-Werk eine kompositorische Praxis. Dennoch weitet sich das interpretative Wirkungsspektrum aus. Einmal stellt das Poly-Werk für den Interpreten eine zusätzliche Herausforderung dar, da durch die verschiedenen Werkkontextualisierungen auch die Absolutheit einer Interpretation relativiert wird und eine Flexibilität erforderlich macht. Weitet man aber auch den Begriff des Interpretativen an sich, dann kann man außerdem sagen, dass der Komponist durch die verschiedenen Kontextualisierungsmöglichkeiten selbst ein Interpret seines Werkes wird.

102 Siehe beispielsweise Cage: Für die Vögel, S. 95, S. 184 f.; „Lecture on Something“, in: ders.: Silence. Lectures and Writings by John Cage, S. $129 \mathrm{f}$.

103 Eco, Das offene Kunstwerk, S. 56 . 
Cages Konzept eines „Musicircus“ wurde bereits 1753 von Joseph Haydn vorweggenommen. Haydn hatte den Einfall, mehrere Musiker am Tiefen Graben in Wien vor den Häusern und in den Ecken zu einem „Gassatim“-Konzert zu versammeln. Jeder Musiker war aufgefordert, das zu spielen, was er wollte. Das Konzert erregte damals schnell den Unmut der Anwohner und endete mit der Verhaftung des Paukers und eines Geigers. ${ }^{104}$ Olga Neuwirth beschreibt dieses Ereignis aus heutiger Perspektive als anarchisch, provokativ und herausfordernd und als Vorgriff auf die Schichtungsverfahren bei Charles Ives, ${ }^{105}$ von dessen Vater, dem Kapellmeister George Ives, ebenfalls überliefert ist, dass er einmal zwei Blaskapellen aneinander vorbeiziehen ließ und sich an den aufeinanderprallenden Klängen freute. ${ }^{106}$ Die Anekdote erinnert ebenso an Mahler, der einmal auf einem Jahrmarkt die durcheinanderklingende Klangkulisse als Polyphonie bejubelt haben soll. Von anderen Komponisten ist Ähnliches überliefert. Den jungen Haydn scheint die Aussicht auf ein unkalkulierbares mehrstimmiges Spektakel, das einer ordnungsstiftenden Kraft entbehrte und sich dem fixen Rahmen des institutionalisierten Konzertbetriebs entzog, jedenfalls schon damals begeistert zu haben. Derlei Aktionen im öffentlichen Raum greifen in einer sehr direkten und unmittelbaren Form die Lebendigkeit des akustischen Nebeneinanders der Straße, der Marktplätze oder anderer öffentlicher Plätze auf. Die Piazza San Marco ist dafür berühmt. Hier spielen drei Kapellen gleichzeitig verschiedene Stücke und Programme. Die Gleichzeitigkeit nimmt man am besten von der Mitte des Platzes aus wahr.

Wohnte Aktionen dieser Art früher ein subversiver Akt der Rebellion inne, haben in jüngster Zeit Veranstalter die Simultanaufführung für großformatige und aufsehenerregende monumentale Konzertevents entdeckt. An die Stelle von Spontanität und Unverbindlichkeit tritt nun eine präzise dramaturgische Planung im Vorfeld. 2016 präsentierte das Festival Wien Modern unter der Mitwirkung von fünfzehn Streichquartetten sämtliche Streichquartette von Schostakowitsch simultan und sich gegenseitig überlappend in einem Konzertsaal. ${ }^{107}$ Die sich zeitlich überlagernde Aufeinanderfolge wurde im Vorfeld genau kalkuliert (vgl. Abb. 1).

104 Siehe auch Howard C. Robbins Landon: Haydn. chronicle and works, in five volumes, London: Thames and Hudson, 1976-1980, Bd. I, S. 64.

105 Siehe http://www.olganeuwirth.com/text26.php (letzter Aufruf am 13.3.2020).

106 Siehe Alex Ross: The Rest is Noise. Das 20. Jahrhundert hören, München: Piper, 2009, S. 153.

107 Siehe auch https://www.mdw.ac.at/schostakowitsch/ (letzter Aufruf am 13.3.2020). 


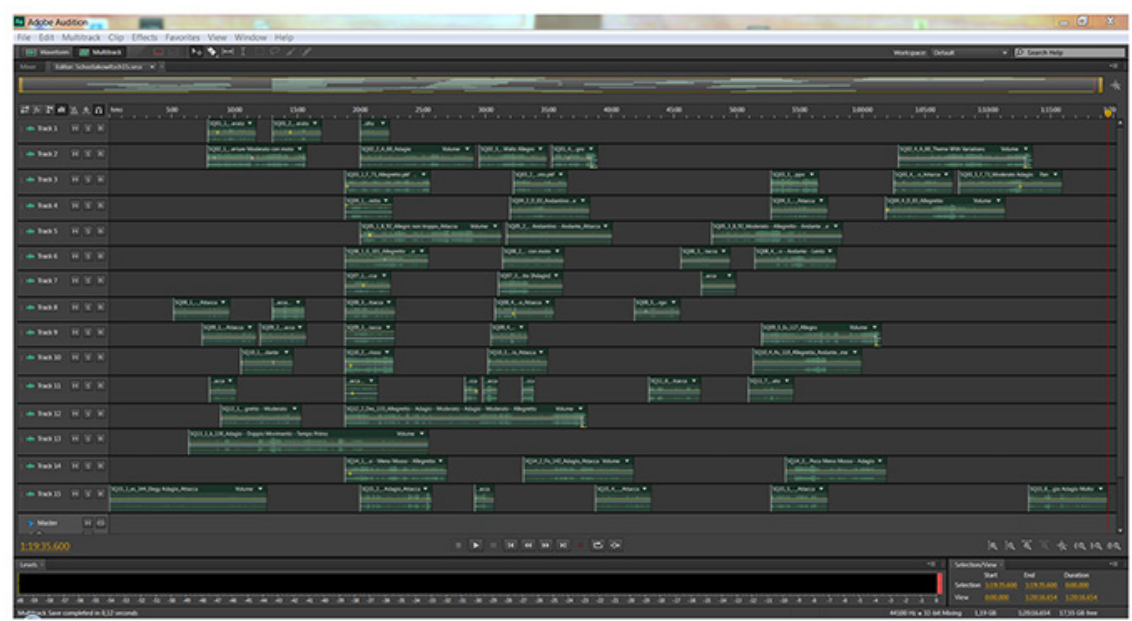

Abb. 1 Übersicht über die zeitliche Verteilung der fünfzehn Schostakowitsch-Quartette über den Gesamtablauf. Screenshot der Audiosimulation. Mit freundlicher Genehmigung von Wien Modern. ${ }^{108}$

Der technische Stand macht es möglich, auch räumlich weit auseinanderliegende Aufführungsorte miteinander zu verbinden, die verschiedenen Aufführungen perfekt zu synchronisieren und an einem Ort simultan für ein Publikum erlebbar zu machen. Zum 30o-jährigen Jubiläum der Stadt Karlsruhe fand 2015 ein Orgelwerk mit dem Titel Organum von Wolfgang Mitterer als Simultankonzert seine Uraufführung. Das Spiel von acht Organisten verschiedener Kirchen in Karlsruhe und den Partnerstädten wurde über eine Satellitenverbindung zeitgleich in den Lichthof der Hochschule für Gestaltung übertragen und dort in einer für die Zuhörer begehbaren Klang- und Videoinstallation zusammengeführt. ${ }^{109}$ Die einzelnen Stränge wurden von Mitterer auf dieses Ereignis hin konzipiert. Sie übersteigen daher den Begriff einer Simultanaufführung im Sinne einer reinen Aufführungspraxis. Viel wichtiger ist hier der Aspekt der telematischen Performancepraxis, die mittels der sich immer weiter entwickelnden Kommunikationstechnologien auch immer besser synchronisierte und inszenierte Performances hervorbringt. Es liegt auf der Hand, dass die dadurch mögliche Überwindung großer räumlicher Distanzen, die unterschiedliche Räume an einem Ort zusammenführt,

108 Aus dem „Letter and Overview of Cues“, unveröffentlichtes Dokument zum Konzertablauf (Version 25.10.2016).

109 Siehe auch https://www.film-tv-video.de/productions/2015/11/14/simultankonzert-achtorgeln-ein-werk-acht-organisten/ (letzter Aufruf am 13·3.2020). 
zukünftig auch für die Simultanaufführung programmatisch sein wird. Die Zusammenführung verschiedener historischer Zeitspannen zu einer Zeit findet in der Zusammenführung weit voneinander entfernter Räume an einem Ort ihre Fortsetzung.

So erregte in der Theaterwelt beispielsweise die Simultanaufführung Die Parallelwelt des Regisseurs Kay Voges, die 2018 am Berliner Ensemble und dem Schauspiel Dortmund ihre Premiere erlebte, großes Aufsehen. In der Aufführung spielen zwei Ensembles zeitgleich den Lebensweg eines Menschen in umgekehrter Abfolge: Hier setzt das Stück mit der Stunde der Geburt, dort mit der des Todes ein. Beide Bühnen sind über einen Videostream miteinander verbunden. Die reale Bühne präsentiert sich als realer Nebenraum zur Videoleinwand, die die Inszenierung aus der anderen Stadt zeitgleich überträgt. Beide Bühnen sind so für den Zuschauer parallel sichtbar. Der Verlauf des Theaterabends ist vom Nebeneinander beider Erzählstränge geprägt, die sich in der Lebensmitte zum Hochzeitsereignis (dem Moment der Vereinigung) überkreuzen. Plötzlich kommt es zu Interaktionen, Verwicklungen und Verwirrungen zwischen den Darstellern im Hier und Dort. Das Theaterstück existiert lediglich als Simultanversion. Die beiden Hälften für sich genommen wurden nicht einzeln aufgeführt. Voges bezieht sich außer auf eine Reihe von philosophischen, naturwissenschaftlichen und gesellschaftlich-sozialen Phänomenen und Gegebenheiten unter anderem auf den von Ernst Bloch (1885-1977) geprägten Begriff der "Gleichzeitigkeit des Ungleichzeitigen“, der für das parallele Nebeneinander und die Überkreuzung verschiedener Lebensstile und Weltanschauungen sowohl in der Gesellschaft als auch im einzelnen Individuum steht. ${ }^{110}$

Auch in der Popkultur gibt es im Genre des Mashups und der aus Coverversionen hervorgegangenen Remixkultur zahlreiche Beispiele für Fusionen einzelner Poptitel und -alben. Eines der bekanntesten Beispiele stammt von Danger Mouse (Brian Burton), der die A-cappella-Versionen des Black Album (2003) von Jay Z mit bearbeiteten Instrumentalspuren des White Album (1968) der Beatles zum Grey Album (2004) verband. Meistens werden im Titel des dritten neuen Songprodukts die ursprünglichen Interpreten durch „vs.“ (= versus) miteinander verbunden, also beispielsweise Jay Z vs. Beatles. Dadurch drückt sich bereits die intendierte „bipolare Konfrontation zweier Künstler“111 aus.

110 Siehe Programmheft zur Aufführung, 2018.

111 Frédéric Döhl: Mash Up in der Musik. Fremdreferenzielles Komponieren, Sound Sampling und Urheberrecht, Bielefeld: Transcript, 2016, S. 81. 
Beim Basic Mashup handelt es sich immer um ein Sampling einzelner isolierter Samples oder Spuren des Ganzen. In der Regel ist das Mashup ein Mix einer vokalen Tonspur des einen Songs mit der instrumentalen Tonspur eines anderen Songs, wobei beide Spuren auch bearbeitet werden können. Die vokalen Tonspuren sind oft Raps, was die harmonische Zusammenführung beider Songs leichter macht.

Demgegenüber rekontextualisiert der Zürcher Künstler Peter Tillessen unter dem Motto Whatever ${ }^{12}$ tatsächlich die kompletten Popsongs, indem er sie als Cover Mashup simultan übereinanderschichtet.13 Dafür wurden 3400 Songs der Rock- und Popgeschichte analysiert, in eine Datenbank eingeführt und auf ihre harmonischen Gemeinsamkeiten hin ausgewertet. Ausgewählt wurden Songs, die dasselbe harmonische Gerüst teilen und sich aufgrund der harmonischen Übereinstimmungen für eine Songpaarung eignen. Beispiele sind Frankie von Connie Francis (1959) kombiniert mit I was the one von Elvis Presley (1956), Let it be (1969) von den Beatles und Bob Marleys Three Little Birds, Stay von Maurice Williams (1960) und The Last Kiss von Pearl Jam (1961), So lonely von The Police (1978) und With or without you von U2 (1987). Durch die harmonisch-strukturellen Übereinstimmungen ergänzen sich die ursprünglich einander fremden Songs zu einer reizvollen Duokonstellation. Der differente Höreindruck der Songpaare, die Clemens Krümmel als "musikalische Vexierbilder" bezeichnet, ${ }^{114}$ entspringt vor allem aus der Vertrautheit mit den akustischen Originalen und entsteht in dem spezifischen Transitraum, der zwischen den beiden Singles angesiedelt ist. Trotz des kontrolliert-systematischen und auf Harmonie und Konsens ausgerichteten Auswahlverfahrens öffnet sich im Dazwischen der vertrauten Songstrukturen ein Raum für Zufallselemente und Unvorhersehbares, der aus dem nichtkalkulierten Aufeinanderstoßen der verschiedenen Melodien, Rhythmen und Texte hervorgeht. Tillessen knüpft mit Whatever (engl. = was auch immer) explizit an die Tradition des in der Renaissancezeit entstandenen und im Barock beliebten Quodlibets (lat. = wie es beliebt) an, bei dem ebenfalls verschiedene Lieder mit identischen harmonischen Akkordfolgen simultan kombiniert werden konnten. Ein bekanntes Beispiel aus dieser Zeit ist Ludwig Senfls Kombination der Volkslieder Ach Elslein, liebes Elselein und Es taget vor dem Walde aus dem Jahre 1544. Es gibt viele andere Beispiele auch

\footnotetext{
112 Siehe https://www.tillessen.info/whatever (letzter Aufruf am 13.3.2020).

113 Eine Darstellung des Projekts aus verschiedenen Perspektiven bietet der Band Whatever. Über doppelte Musik, hg. von Tania Prill und Peter Tillessen, Leipzig: Spector Books, 2020.

114 Clemens Krümmel: „Der Popsong und sein Double. Zu Peter Tillessens Quodlibets“, in: Whatever, S. $45^{-} 53$, hier: S. $5^{2}$.
} 
für Kanonquodlibets, die verschiedene Kanons parallellaufen lassen. Gerade an bekannten Beispielen mit einer tonalen Basis lassen sich durch simultane Überlagerungstechniken, die den harmonischen Verlauf berücksichtigen, reizvolle Kontextualisierungen erzeugen, die das einst Vertraute in einem neuen Licht erscheinen lassen können.

Tillessen dachte die Idee noch weiter und schichtete in Algo sowohl alle Präludien als auch alle Fugen des Wohltemperierten Klaviers von Johann Sebastian Bach übereinander. Die dabei entstehende auf Vermassung und Verdichtung ausgerichtete Klangtotale, in der die einst individuellen Fugencharaktere vom Klangkontinuum vollständig absorbiert werden, lässt sich treffenderweise als jene „vibrierende ,Nicht-Figur' ' beschreiben, die John Cage im Gespräch mit Daniel Charles ${ }^{115}$ beschwört (vgl. Kapitel 1.3.3) und die aus ebenjenem Umschlag der Linearität in die Nichtlinearität hervorgeht.

Die Möglichkeit der wirklich "massenhaften“ Akkumulation präexistenter Songs und Alben wurde von diversen Komponisten und Künstlern elektroakustischer Musik und der Konzeptkunst recht ausgiebig betrieben. Im Mittelpunkt steht hier der Umschlag der Musik und der Werke in das Stadium des Rauschens. Der Komponist Marko Ciciliano verarbeitete in seinem Pop Wall Alphabet 26 Alben der Popmusikgeschichte nach einer einheitlichen Systematik, indem er sämtliche Songs eines Albums sowie ihre spektralen Freezes in einem zweiteiligen Prozess des Ab- und Aufbaus zu einem NoiseBand übereinanderschichtete. Das Verfahren ist für alle Alben gleich. Die Qualität des resultierenden Noise-Bands ist aber albumspezifisch und sowohl von musikalischen Parametern als auch den akustischen Parametern der Studioarbeit geprägt.

In der musikalischen Konzeptkunst gibt es seit den 7oer Jahren mehrere Beispiele von Werken, für die bekannte Meisterwerke der klassischen Musik zum Rauschen aufaddiert wurden und sich gegenseitig annullieren. Ihre Transposition in den Status einer reinen Materialschicht geht mit einer Neutralisierung ihrer Charakteristika einher und macht das Material offen für den folgenden Transformationsprozess: Aus horizontal-geschlossenen Werkverläufen werden statisch-vertikal kondensierte Klangobjekte. Deren neue Charakteristika, sei es als akustisches Rauschband oder als Klangsäule, sind primär von den verwendeten Verfahren der Überlagerung und sekundär von der Wahl der verwendeten Musikwerke geprägt. Der Prozess vom Werk

115 Siehe Cage: Für die Vögel, S. 251. 
zum Klangobjekt markiert einen qualitativen Umschlag in der Form- und Werksubstanz. ${ }^{116}$

Der Komponist Peter Ablinger $\left({ }^{*}\right.$ 1959) zerlegte in den 8oer Jahren im Studio alle neun Symphonien von Beethoven in 40-sekündige Abschnitte (Weiss/ Weisslich 22) und überlagerte diese simultan zu einem Rauschen. Dasselbe machte er mit den Symphonien von Haydn, Mozart, Beethoven, Schubert, Bruckner und Mahler. Sechs- bis achtstündiges symphonisches Material wird in 40-Sekunden-Einheiten geteilt, und diese Einheiten werden simultan überlagert. Diese Arbeit ist Teil des Zyklus Weiss/Weisslich, der sich in verschiedenen Studien mit den unterschiedlichen Klangqualitäten des Rauschens auseinandersetzt. Ablinger bezog sich mit dieser Arbeit auf eine Aussage von John Cage, ${ }^{117}$ der die Frage, ob er sich vorstellen könnte eine BeethovenSymphonie zu dirigieren, bejaht hatte unter der Voraussetzung, alle gleichzeitig dirigieren zu können. ${ }^{118}$ Cage wiederum verweist in einer Fußnote zu dieser Aussage ${ }^{119}$ auf den Komponisten Hal Freedman, der im Elektronischen Studio der Yale-Universität 1974-1975 in Ring précis alle Teile des Ring-Zyklus von Wagner und in einer weiteren Arbeit Précis 2 alle Streichquartette von Bartók übereinanderlegte. ${ }^{120}$

Ein weiteres radikales Beispiel simultaner Überlagerung unternahm der Komponist Nick Fells, der für das Stück Two - Capriccio aus Other Islands ${ }^{121}$ verschiedene Capriccios von Paganini übereinanderschichtete. Mithilfe eines Bildbearbeitungsprogramms legte Fells die einzelnen Capriccios in einer Bilddatei exakt übereinander, arbeitete dabei aber mit unterschiedlicher Deckkraft innerhalb der Graustufenskala. Die Notation wird angenähert an

116 Auch die Clusterbretter. Objekte für ein Klavier (1996) der Künstlerin Dodo Schielein fallen in diese Kategorie. Die Clusterbretter umfassen die Länge einer Klaviatur und können alle vorkommenden Töne einer von Schielein ausgewählten bekannten Komposition anschlagen, zum Beispiel des ersten Satzes der Pathetique von Beethoven. Je nach Kompositionsvorlage unterscheiden sich die resultierenden Clusterklänge in ihrer Farbe und Anschlagsform (das Debussy-Brett wird beispielsweise arpeggiert angeschlagen). Im Werkschaffen von Robert HP Platz findet sich auch eine vertikale Kondensation, die als einzelne Klangsäule den horizontalen Werkverlauf des Orchesterstücks Weiter in einem zweiten Orchesterstück Turm, das aus einem einzigen 4/4-Takt besteht, extrem verdichtet. Hier liegt allerdings kein technisch neutrales Verfahren zugrunde, sondern eine kompositorische Eigenbearbeitung.

117 Peter Ablinger: „Das Alte im Neuen. Über den Umgang mit musikalischer Weltliteratur in der Musik des 2o. Jahrhunderts“, Radiovortrag vom 7.12.1991, Deutschlandsender Kultur, https://ablinger.mur.at/docs/altneu.pdf (letzter Aufruf am 27.4.2020).

118 Siehe Cage: Für die Vögel, S. 113 .

119 Ebd.

120 Diese Arbeit erschien bei dem Label Opus One 1983 als LP.

121 Other Islands ist für Sopransaxophon, Violine, Klavier und Elektronik geschrieben. 
ein „bildliches Rauschen“, auf das der ausführende Musiker individuell zu reagieren hat (vgl. Abb. 2).
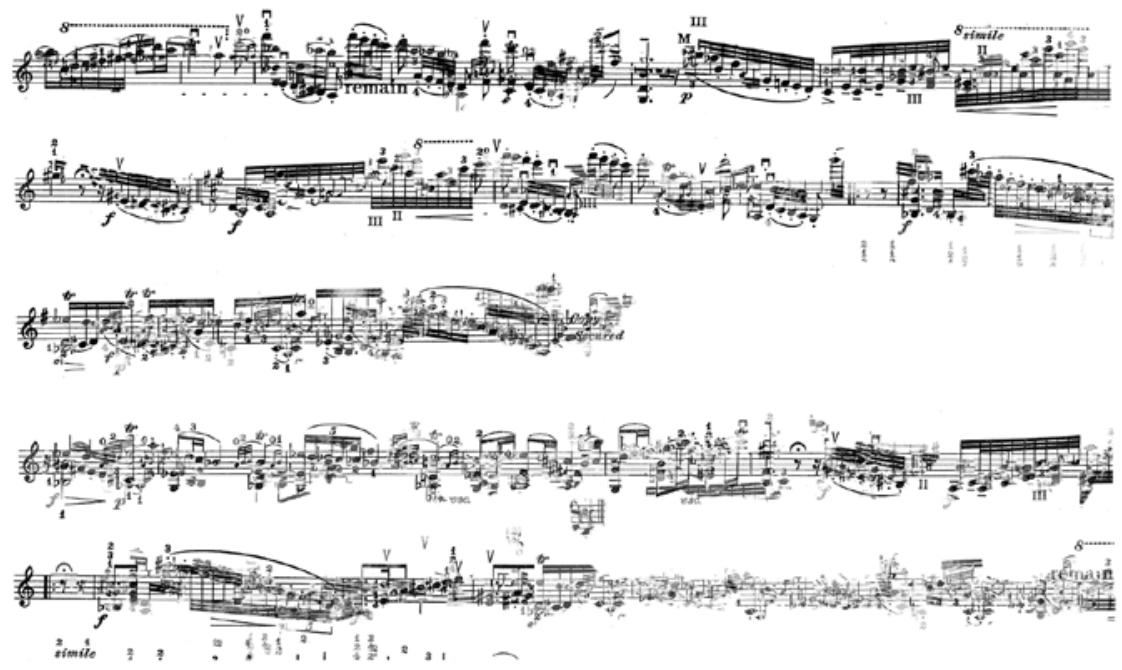

Abb. 2 Nick Fells: Other Islands (Ausschnitt) für Violine, 2009. Simultane Überlagerung verschiedener Capriccios von Paganini. Mit freundlicher Genehmigung des Komponisten. 